\title{
Generalized network modeling: Network extraction as a coarse-scale discretization of the void space of porous media
}

\author{
Ali Q. Raeini, Branko Bijeljic, and Martin J. Blunt \\ Department of Earth Science and Engineering, Imperial College London, London SW7 2AZ, United Kingdom
}

(Received 31 March 2017; published 20 July 2017)

\begin{abstract}
A generalized network extraction workflow is developed for parameterizing three-dimensional (3D) images of porous media. The aim of this workflow is to reduce the uncertainties in conventional network modeling predictions introduced due to the oversimplification of complex pore geometries encountered in natural porous media. The generalized network serves as a coarse discretization of the surface generated from a medial-axis transformation of the 3D image. This discretization divides the void space into individual pores and then subdivides each pore into sub-elements called half-throat connections. Each half-throat connection is further segmented into corners by analyzing the medial axis curves of its axial plane. The parameters approximating each corner - corner angle, volume, and conductivity — are extracted at different discretization levels, corresponding to different wetting layer thickness and local capillary pressures during multiphase flow simulations. Conductivities are calculated using direct single-phase flow simulation so that the network can reproduce the single-phase flow permeability of the underlying image exactly. We first validate the algorithm by using it to discretize synthetic angular pore geometries and show that the network model reproduces the corner angles accurately. We then extract network models from micro-CT images of porous rocks and show that the network extraction preserves macroscopic properties, the permeability and formation factor, and the statistics of the micro-CT images.
\end{abstract}

DOI: 10.1103/PhysRevE.96.013312

\section{INTRODUCTION}

Pore-scale modeling has wide applications in petroleum engineering, hydrology, and environmental engineering $[1,2]$. With advances in micro-CT imaging and the resultant availability of high-resolution representations of porous media [3], pore-scale models are now widely used as a tool to obtain macroscopic parameters, such as relative permeability and capillary pressure curves, and dispersion and reaction rate coefficients. These macroscopic parameters, in turn, are used as input to larger-scale models for predicting the field-scale behavior [4].

To capture the complex geometry of real materials more accurately, a variety of direct methods have been used to study single and multiphase flow through porous media at various scales [5]. These include molecular simulations studying fluidrock interactions over nanometers and mesoscale simulation methods, such as lattice Boltzmann and the finite volume method, that can be used to solve single- and two-phase flow equations directly on three-dimensional (3D) images of porous rocks. Direct single-phase simulations can be performed in a practical computational time to obtain absolute permeability and electrical properties over a representative elementary volume in homogeneous samples [6,7]. Direct two-phase flow simulations, however, are computationally more expensive and limited to small image sizes and high capillary numbers, typically for capillary numbers higher than $10^{-6}$ and image sizes around $400^{3}$; these simulations require high-performance computing and may take many days of computational time [8-10].

Reservoir rocks typically contain pore sizes that span four orders of magnitude, from submicron microporosity to millimeter-sized vugs in carbonates, for instance. A representative elementary volume, from which robust macroscopic properties may be computed, such as absolute and relative permeability, may need to be some tens of the largest pores across. Then, at the smallest scales, to capture features such as wetting layers and the complex displacement dynamics, every pore needs to be discretized — in a direct simulation - by at least an order of 10 grid blocks or particles. In many systems, six orders of magnitude, or more, may therefore be required from the size of the cells within the smallest pores to the overall size of the system, or $10^{18}$ blocks in total in three dimensions, to capture multiphase flow over a sufficient number of pores to compute averaged properties adequately [1]. This is an impossible task, even with high-performance parallel computing, and is akin to the related problem in field-scale simulation where it is not possible to resolve the heterogeneity down to the centimeter-scale of direct laboratory measurement. Indeed, the current fixation with using direct simulation to compute averaged properties is unhelpful and unlikely to be generally successful. What is required is a hierarchy of approaches combined with upscaling. This is well accepted in the transition from molecular dynamics to averaged equations-Navier-Stokes and Young-Laplacewithin the pore space: the same concept needs to be applied to average flow and displacement within a pore to find, for instance, conductance and capillary entry pressure, that are then input into larger-scale simulations capable of computing displacement over many thousands, or millions, of pores.

In this respect a network model is simply a coarse representation of the flow domain (3D image). The network elements follow the pore-space geometry, in the same way that a large-scale geological model has grid blocks that capture the main stratigraphic surfaces [11], and have properties assigned to them-conductance, volume, and capillary pressure-that allow larger-scale computations to be made.

This paper demonstrates how to embrace this philosophy, applied to pore-space images of rock samples, using direct simulation as the first step in a pore-to-core upscaling procedure. Rather than the current absurd view of direct simulation and network modeling as being rival methods, they instead 
complement each other, allowing an accurate representation of multiphase flow over many orders of magnitude in length scale.

Pore-network modeling divides the void space of the rock into pores representing wider regions that are connected through narrower restrictions called throats. This representation is then used to model flow by computing and tracking the pore-scale configuration of fluid phases for different displacement sequences using empirical equations derived normally from semianalytical expressions for pores of a simple geometry. This approach has proved successful for the study of various phenomena, such as interfacial area and capillary pressure [12-14], reaction [15,16], drying [17], evaporation [18], dissolution [19,20], electrical properties [21-24], nonNewtonian flow [25,26], foam flow [27], solution gas drives [28,29], gas condensate systems [30-32], water vapor transport [33], dispersion [34-36], and two- and three-phase flow [3742]. However, for pore-network models to have any predictive power for studying flow through the full range of samples encountered in geological settings, an accurate parametrization of the void space is needed.

\section{Conventional pore network models}

The first network models based on an explicit representation of a porous medium were presented by Bryant and colleagues [43-45]. They extracted networks from a random close packing of equally sized spheres [46]. They were able to reproduce the absolute and relative permeability, capillary pressure, and electrical and elastic properties of water-wet sand packs, sphere packs, and a cemented quartz sandstone, and to match the trend of permeability with porosity. Øren, Bakke, and coworkers at Statoil extended this work and developed a reconstruction method, where the packing of grains of different size was simulated followed by geological processes such as sedimentation, compaction, and diagenesis $[47,48]$. They used the geological reconstructions to extract topologically equivalent networks through which multiphase flow was simulated. Another approach is the use of statistical models to generate synthetic 3D structures that capture the properties of two-dimensional (2D) thin sections [49-55].

The advent of 3D imaging, usually using x-ray microtomography with resolutions of around $1 \mu \mathrm{m}$, has enormously increased the availability of pore-space images of rocks of interest $[3,56]$. In such images, grain identification may be challenging, particularly for carbonates and heavily cemented samples, and so a more topological approach is required to extract an equivalent network. These methods are based on finding pores in regions that can accommodate the largest spheres, called maximal balls [57-61], with throats at restrictions, that can be identified from the medial axis skeleton of the pore space $[62,63]$. The topology of the pore network using these methods is related to the watershed segmentation of the distance map [1,64] - the distance to the nearest solid surface. Filtering is needed to avoid the sensitivity of the algorithm to surface roughness [47,54,65-68]. The watershed approach divides the void space into pore regions where the distance map increases to a pore center: throats are surfaces of minimal distance separating two pore regions. These methods of network extraction have produced reasonable representations of the pore space and have been used to successfully reproduce multiphase flow properties for a variety of rock types [59,62,69-71].

In general, however, the focus of these studies has been on an explicit topological construction of a network, where the properties to be assigned to network elements-pores or throats-are based on specific geometrical assumptions on the shape of the elements. Conventionally, intermediate parameters such as radius $(R)$, shape-factor $(G$, ratio of area to perimeter squared), volume $(V)$, area $(A)$, and length $(L)$ are used to describe individual network elements. These parameters are used during flow simulation to compute fluid volumes, conductivities, and fluid-fluid interfacial curvatures. Then the flow properties are averaged (upscaled) to compute the fluid saturations, effective permeabilities, and capillary pressures of the whole network. However, the assignment of a boundary between pores and throats-hence the definitions of pore and throat lengths and volumes [72]—is arbitrary. Moreover, a shape factor does not uniquely define a geometry, even for simple shapes. Therefore, the values assigned to the flow properties, deduced from the intermediate parameters $(G, R, V, A, L)$, may not provide a good representation of flow through the original pore space image and may produce significantly different results depending on the algorithms used to calculate them [73].

\section{NETWORK EXTRACTION AS A DISCRETIZATION OF THE PORE-SPACE}

The generalized network model serves as a coarse discretization of the void-space image into pore-sized elements and their parametrization. In this paper, we discretize the void-space into pores and further into half-throat corners as illustrated in Figs. 1(a) and 1(b). During this transformation, each voxel of the void-space is assigned to a unique pore, a unique half-throat, and a unique corner. The parameters describing each corner are extracted directly from the original image, using a medial-axis transformation of the void space and direct single-phase flow simulations at different discretization levels [see Figs. 1(c) and 1(d)].

The medial axis transform is defined as the set of points equidistant from two or more points on the solid boundary. The distance to the nearest solid boundary is also recorded. The medial axis transformation can be applied to 3D images to produce a set of isolated points (for the case of isolated spherical pores), curves (for the case of throats with circular cross-section), and surfaces [63,74,75]. Medial axis transformation can be applied to cross-sections of the half-throats in their axial plane (see Fig. 1), which leads to medial axis curves and can be used to identify throat corners directly from the image. In this paper, we refer to the medial axis transform of the 3D pore space simply as its medial surface to avoid ambiguity with the curves obtained from medial-axis transformation of throats' cross-sectional areas. The medial axis transform preserves the topology of any $n$-dimensional geometry [76]. Therefore, by preserving the topology of the medial surface during network extraction, we retain the topology of the underlying image. In practice, however, we need to filter the surface roughness while capturing the main features of the void 
(a)

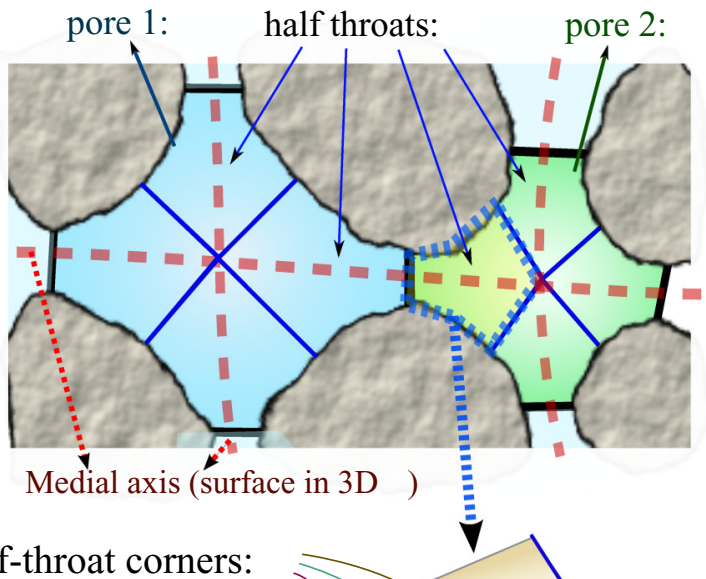

(b)

(c)

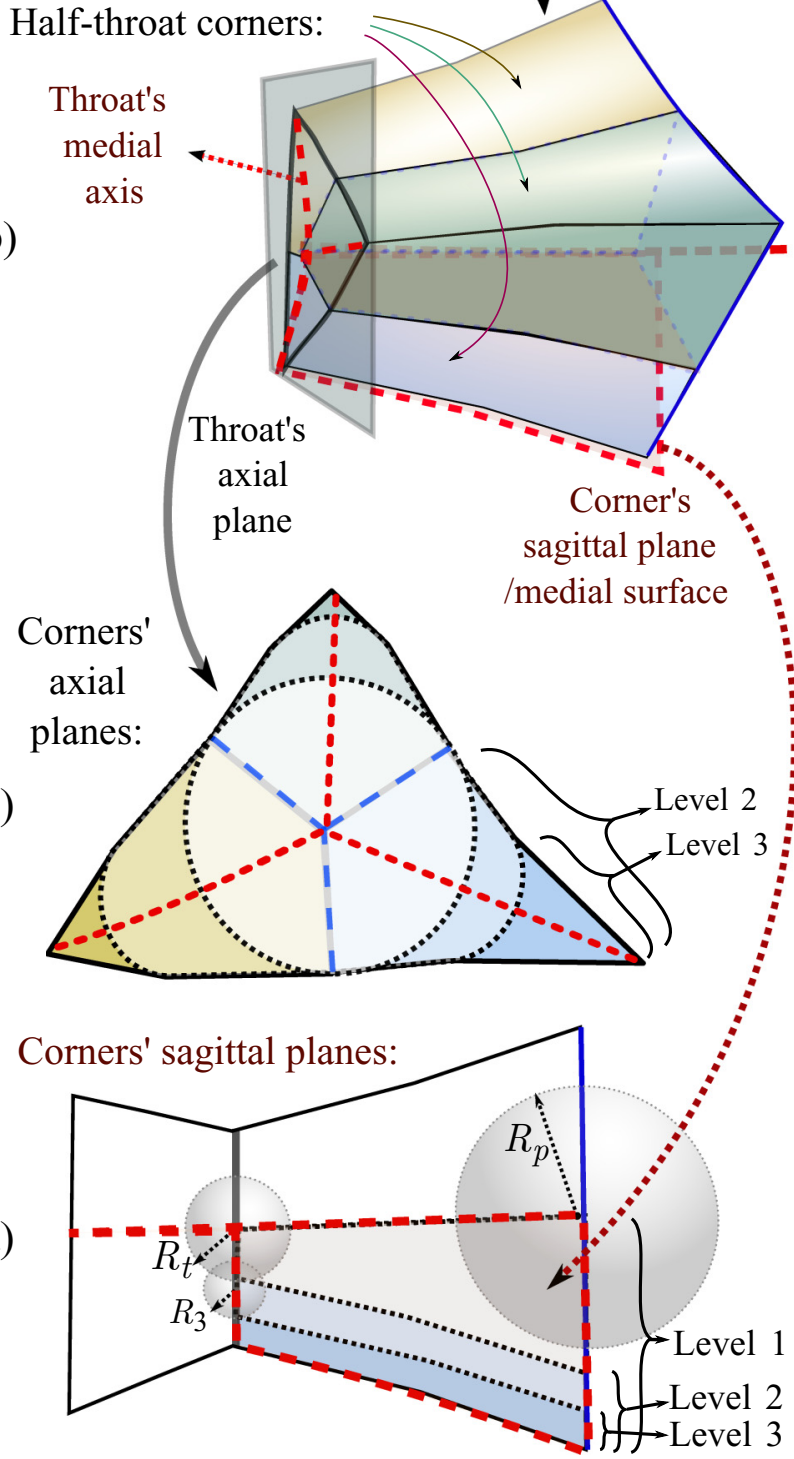

FIG. 1. An illustration of the network extraction workflow: (a) segmentation of void space into pores (separated by thick solid black lines) and subsequently into half-throats (separated by solid blue lines) and (b) into half-throat corners where the network parameters are extracted. (c, d) Illustrations of different levels (separated by dotted black lines) used to discretize the corners (separated by dashed blue lines), in the corners' axial and sagittal planes, respectively. Note that the size of inscribed spheres shown in (d) decreases from the pore center toward the throat center and further toward the corner crevices. space. The effect of surface roughness can be added during flow simulation-for instance, by considering contact-angle hysteresis during contact line motion in two-phase flow.

The division of the void space into pores and throats, which define the connectivity of the network in this work, is, in principle, similar to that of other authors-most importantly, those methods that are now used to provide commercial digital rock analysis services $[47,59,77]$. Similar to these conventional network extraction methods, we use the distance mapwhich is the same as maximal-sphere radius-and medial axis transformation to partition the void space into pores and identify pore-to-pore connections (throats). However, as opposed to conventional network models with largely arbitrary parameters, the boundary between pores and throats and shape-factor, for instance, to describe individual elements, we use a flexible parametrization using half-throat corners, which preserves the subpore scale properties of the void space. While this - at first sight—may appear to be simply a more elaborate geometrical representation of elements, it is needed for a robust characterization of the relationship between volume, capillary pressure, and conductance for any contact angle and displacement sequence. More importantly, the approximation errors associated with the network extraction and flow modeling are minimized by computing the flow conductivities assigned to corner elements using direct simulation of flow on the underlying image.

Once the method has been described, it will be tested on a suite of benchmark images of quarry samples. We take advantage of the one-to-one correspondence between the corner elements in our model and the underlying image to validate the discretization of the pore space in the generalized network model. In a companion paper, these networks will be used to compute multiphase flow properties and the results compared to direct two-phase flow simulations and experimental measurements [78].

\section{NETWORK EXTRACTION WORKFLOW}

The following presents an overview of different stages of the network extraction algorithm.

(1) Calculate the distance map - the distance of each voxel from the nearest solid wall.

(2) Identify pores and throats.

(2.1) Extract medial surface of the void space:

(i) Select an initial set of voxels, one in each $2^{3}$ block of voxels;

(ii) Sort the initial set in order of the distance map;

(iii) Remove voxels with spheres-at the voxel centers with the same radius as their distance map-fully overlapped by bigger spheres;

(iv) Remove voxels which are close to bigger spheres in the set;

(v) The remaining voxels are centered on the medial surface, assign each of them a maximal sphere;

(vi) Refine the location of maximal-sphere centers (medial-surface points) within each voxel.

(2.2) Segment the medial surface:

(i) Generate a maximal-sphere hierarchy: bigger spheres are marked as the parent of nearby partially overlapped smaller spheres; 
distance map (voxels)

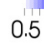

$10 \quad 25$
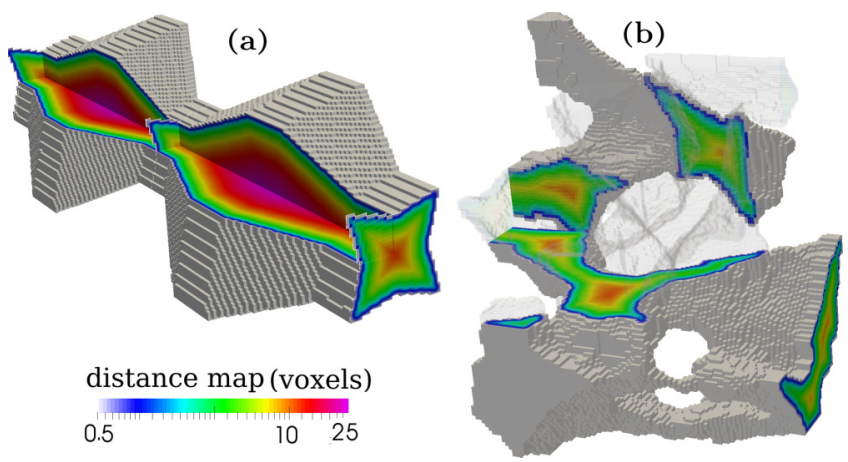

FIG. 2. Distance map computed for each pore voxel for (a) a star-shaped geometry and (b) a small piece of an image of Bentheimer sandstone of size $100^{3}$ voxels, $0.3^{3} \mathrm{~mm}^{3}$, used for illustration of the network extraction algorithm.

(ii) Assign a unique pore labels to maximal spheres with no parent-no bigger nearby sphere.

(2.3) Map pore labels to the underlying 3D imagesegment the image into pore bodies.

(2.4) Collect voxel faces shared by voxels of different pores to generate throat surfaces-bounding surfaces between neighboring pores.

(3) Segment pores into half-throat corners:

(3.1) Generate medial axes of the throat surfaces;

(3.2) Assign a corner label to each branch of the medial axes;

(3.3) Generate a 3D image of corners: map the corner labels from the throat medial axes branches to the underlying image and grow them toward pore centers.

(4) Parametrize half-throat corners at 3 levels, $i$, associated with inscribed radii $R_{i}=R_{p}, R_{t}, 0.7 R_{t}$ :

(4.1) Remove voxels in the center of the void space, which are inside spheres with radius greater than $R_{i}$, from the corner image;

(4.2) Run single-phase flow simulation (DNS) on the remaining voxels for computing corner conductivities;

(4.3) Compute corner conductivities, volume, and cross sectional areas, for each level.

(5) Export corner parameters and network connectivity information as input to network flow simulator.

Each of these stages are described in more detail in the following sections.

\section{Stage 1. Calculation of the distance map}

The distance map $\left(r_{v}\right)$ is computed at the center of each void voxel (see Fig. 2). It is approximated as the distance of the voxel's center to the center of the nearest solid voxel minus half the voxel length. A direct, but efficient, search algorithm is developed to find the nearest solid voxel. In this algorithm we record the location of the nearest solid voxel obtained for each void voxel and narrow the search region for its neighboring void voxels, to a spherical shell with a width of only one voxel rather than a full sphere.
Stage 2. Medial-surface extraction and watershed segmentation of the distance map to generate pores and throats

An unstructured 2D medial-surface mesh-a discretized set of points and their connectivity (the list of neighboring points of each point) — can represent the void space accurately with far fewer points than the number of voxels in the original segmented 3D image. Therefore, it improves the efficiency of the network extraction significantly while at the same time allowing a richer analysis of the pore structure than conventional network models.

To extract the medial surface, we first search for and select a set of voxels on the medial-surface. The selected set of voxels are used to define the medial surface points, which are initially set to be the same as the voxel centers but then refined for subvoxel accuracy. A radius, which is initially the same as the distance map of the voxel, is associated to each of the points. We refer to each point and its associated radius as a maximal sphere, since they fully describe a sphere analytically. We then represent the connectivity of the medial surface using a maximal-sphere hierarchy [59,61]. A maximal ball as used in Dong and Blunt [59] is the largest sphere centered on a voxel that can fit inside the pore space; hence, it has the same radius as the distance map. However, here we only consider maximal balls centered on the medial surface - that touch the solid at two or more points - and refer to these as maximal spheres. The maximal-sphere centers define the medial surface points and their hierarchy defines the connectivity of the medial surface mesh.

The location of the points on the medial surface are refined by moving them from the voxel center by up to 0.5 voxel lengths in the direction of smaller values of the gradient of the distance map, computed using the finite-difference method at voxel faces. This refinement does not relocate the points to a new voxel but it helps to reduce artifacts introduced due to the voxelized (stair-case) representation of the original image and consequently helps in the identification of corner elements in the subsequent steps. The radius of the maximal sphere $\left(r_{\mathrm{ms}}\right)$ is assumed to be the same as the voxel distance-map plus the distance it moved during this refinement.

The maximal spheres on the medial surface cannot be fully overlapped by a bigger maximal sphere; we use this criterion to select the set of voxels to which the maximal spheres are assigned. This is done using an elimination algorithm as follows. First, to save computational time, only one voxel for each eight neighboring voxels, the voxel with the largest distance map, is selected. Furthermore, voxels with a distance map, $r_{v}$, less than a threshold radius are excluded to filter high-frequency roughness on the solid walls. In this paper, this threshold is set to 1.75 voxel lengths. Second, all the selected voxels are sorted to prevent generating spheres that are fully overlapped by bigger maximal spheres and hence not on the medial surface. We then take the voxel with the largest distance map and assign a new maximal sphere ( $m s$ ) to it. All nearby voxels whose maximal spheres will be fully overlapped by $m s$ are deselected. Mathematically this means that $r_{v}+d_{v-\mathrm{ms}}<r_{\mathrm{ms}}$, where $d_{v-\mathrm{ms}}$ is the distance between the centers of the nearby voxel and $m s$. We also exclude voxels close to the center of the new maximal sphere, $m s$, for which $d_{v-\mathrm{ms}}<d_{\mathrm{threshold}, r}\left(r_{v}+r_{\mathrm{ms}}\right) / 2$, to avoid unnecessary details 
(a)
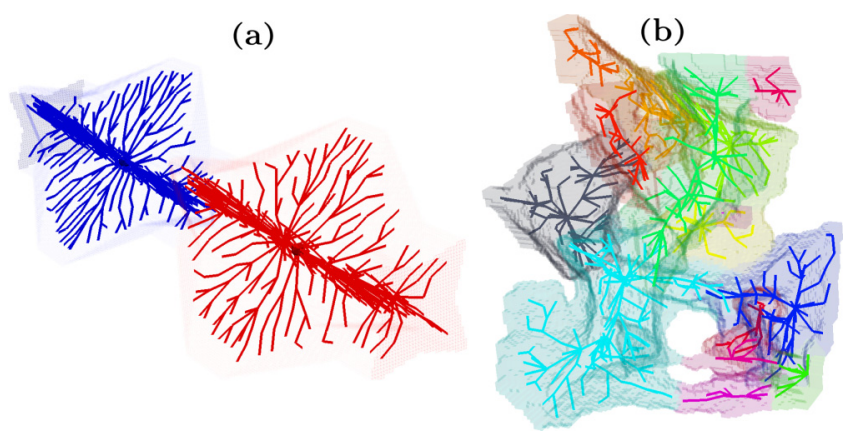

FIG. 3. Medial surface branches (colored lines), generated from connecting centers of the parent-child maximal-sphere hierarchy, for (a) the artificial star-shaped pore space and (b) a section of a Bentheimer sandstone image. The hierarchy generation leads to groups of maximal spheres, which are used to segment (label) the void space into pore bodies (transparent background colors). Different colors show different pore labels.

in the medial-surface discretization. In this paper, we choose $d_{\text {threshold, } r}=0.3$, which was found to lead to an acceptable resolution for the distance between the medial surface points, as shown in Fig. 3. We then repeat this process by taking the next selected voxel with the largest distance map, and so on. In this way, we deselect voxels in the image: we are left with a series of voxels on the medial surface and their associated maximal spheres that provide an approximate representation of the topology of the pore space.

The resulting maximal spheres are grouped using a parentchild hierarchy similar to the maximal-ball algorithm [59]: for each pair of partially overlapping maximal spheres, the bigger sphere is considered as the parent of the smaller sphere. Any maximal sphere that has no neighboring sphere larger than itself (hence, located at the local maxima of the distance map) is assigned as the top level and defines a pore: its center defines the center of the pore and its radius defines the pore radius $\left(R_{p}\right)$. The maximal sphere is given a pore label and this label is also assigned to all children of the maximal sphere. Overall, this leads to a segmentation of the medial surface, as shown in Fig. 3.

We now return to consider all the voxels in the image, to assign every voxel uniquely to a pore (see Fig. 3) and to identify throats. We first map the pore labels of the medial surface points to the original image voxels containing the point. Then we use a region-growing algorithm to extend it to the whole pore space. The region-growing algorithm recursively assigns the pore label of each voxel to its neighboring unassigned void voxels. However, we initially consider neighboring voxels with a smaller distance map. This constraint ensures that the faces where voxels with different pore labels meet have the smallest distance map, hence, consistent with a watershed segmentation of the distance map. We find all the sets of voxel faces where, on either side, the two voxels are assigned to different pores. Each set that separates two adjacent pores defines a throat surface. The voxels on either side of the throat surface are collected and sorted in the order of their distance map, and two maximal spheres are assigned to the voxels with the largest distance map, one for each side. These two maximal spheres are recorded as throat maximal spheres. The throat radius, $R_{t}$, is defined as the average of the radius of these two maximal spheres. A parent-child hierarchy is generated for each of the spheres and the rest of the maximal spheres assigned to the voxels of neighboring pores. The line that connects the center of the throat maximal spheres to the center of its parents, up to the center of the neighboring pore's maximal spheres at the highest level of the hierarchy, is called the throat line.

\section{Stage 3. Segmenting pores into half-throat corners}

To identify the throat corners, a medial axis transformation is applied to the throat surface-a 2D geometry in 3D spaceto generate its medial axis curves-a $1 \mathrm{D}$ geometry in $3 \mathrm{D}$ space. We use a medial axis transformation algorithm similar to the medial surface extraction from the 3D image described at Stage 2. The medial-axis transformation should ideally be applied to the throat surface. However, for the sake of simplicity, we have applied it to the voxels on the side of the throat surface, the side with the larger pore label. First these throat voxels are sorted in descending order of the distance map. Then we assign a maximal sphere to the voxel with the largest distance map. We then exclude all the nearby voxels whose centers are less than 0.3 times the maximal-sphere radius away. As we did for the whole image, we apply this process recursively, assigning maximal spheres to the next voxel with the largest distance map, if the sphere is not fully overlapped by any previously assigned maximal sphere, and so on.

The new maximal spheres assigned to the throat voxels are grouped by generating their parent-child hierarchy: a maximal sphere that partially overlaps a smaller sphere is assigned as the parent of the smaller sphere. The lines connecting the centers of these maximal spheres form medial axis curves that also follow the medial surface branches generated at Stage 2 and extend from the throat center toward the throat corners (Fig. 4), in the throat's axial plane [see Fig. 1(b)].

We assign a corner label to each branch of the throat medial axis curves. The corner labels are mapped back to the original 3D image and are used to segment the whole void space image into half-throat corners using a region-growing algorithm. Initially, the voxels inside the spheres centered on the throat line are excluded from the region-growing algorithm. Starting from the throat surface, the corner labels are successively assigned in all directions into neighboring unallocated void voxels that are not inside the maximal spheres centered on the throat line. The corner label given to a voxel is that of its nearest neighbor. Corner labels are subsequently grown toward throat lines using the same recursive approach and then toward pore centers so that each voxel of the void space is assigned uniquely to a corner, leading a segmentation of the whole void space into half-throat corners that we call, from now on, for simplicity, just corners (see Fig. 4).

\section{Stage 4. Parametrizing half-throat corners}

The network model is considered as a tool for upscaling direct simulations run on the $3 \mathrm{D}$ micro-CT image. The corner parameters are extracted at three discretization levels, $i=1-3$, which are obtained by excluding voxels inside all maximal spheres with radius $r>R_{i}$, where $R_{1}=R_{p}$ (no voxels are excluded), $R_{2}=R_{t}$, and $R_{3}=0.7 R_{t}$. The coefficient 0.7 is 

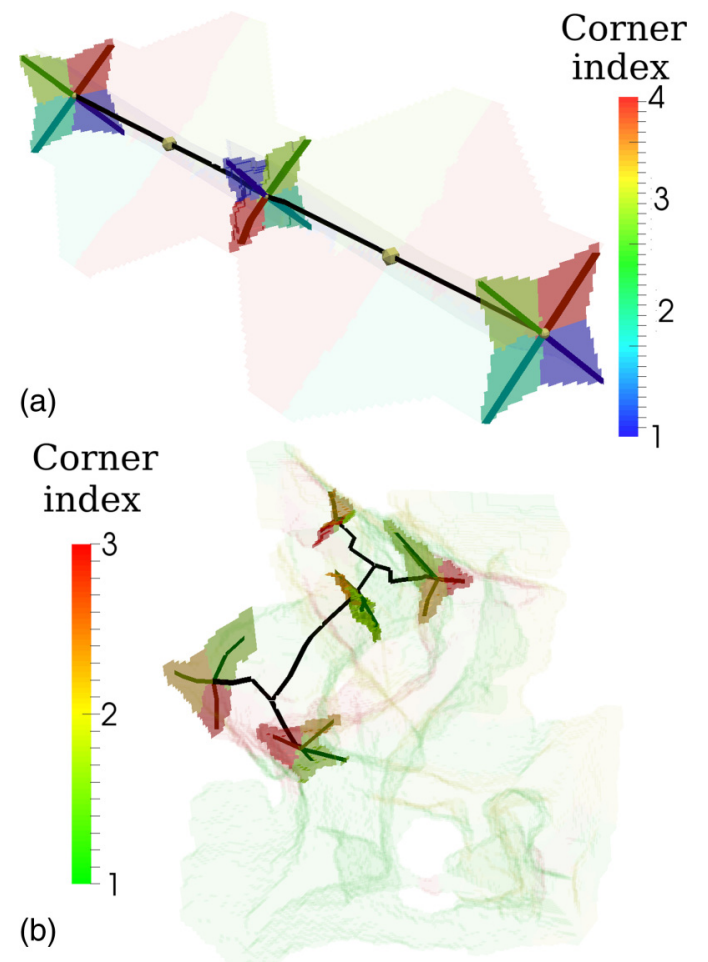

FIG. 4. Medial-axis curves (dark colored lines) of throat surfaces, which are colored by the local corner index, for (a) the star-shaped geometry and (b) two pores of the Bentheimer image. The corner indices are mapped into the underlying 3D image (transparent background colors) using a region growing algorithm as explained in the text. The solid black lines represent the throat lines, connecting throat centers to the pore centers through the maximal-sphere hierarchy.

arbitrary and is chosen so that there will be sufficient voxels remaining in the corner for an accurate evaluation of corner conductivity.

Figure 5 shows visualizations of the corner voxels for the three discretization levels and the computed pressure fields from the solution of the Navier-Stokes equations at a low Reynolds number on the images obtained for each level. The simulations are run on the entire underlying image: we do not compute the flow field of each corner separately. The simulations are performed using the OpenFOAM [79] finite volume library; see Bijeljic et al. [80] and Raeini et al. [81] for details. We have imposed a pressure drop of $1 \mathrm{~Pa}$ between the inlet and the outlet boundaries. No-slip boundary conditions are applied on all other sides of the image, on solid-walls and on the boundary between the voxels considered at a particular discretization level and the excluded voxels. For multiphase flow, this corresponds to a no-flow boundary at fluid-fluid interfaces: the effect of flow velocity and fluid-fluid drag force can be added during network flow simulations [82-84]. These simulations are upscaled to obtain the contribution of each corner to flow conductivity:

$$
g^{q}=\mu Q / \Delta P,
$$

where $\Delta P=P_{p}-P_{t}$ is the viscous pressure drop along the half-throat, where $P_{t}$ is the average pressure of the faces of the (a)
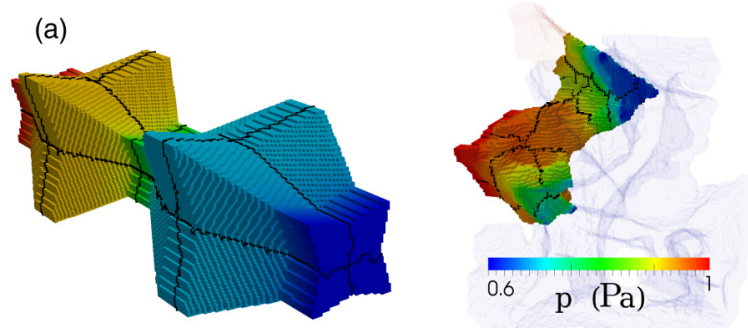

(b)

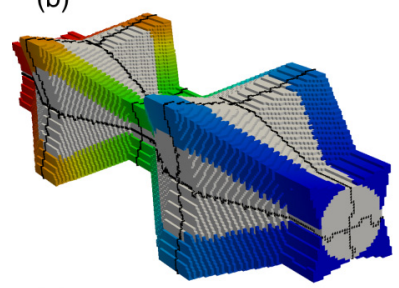

(c)
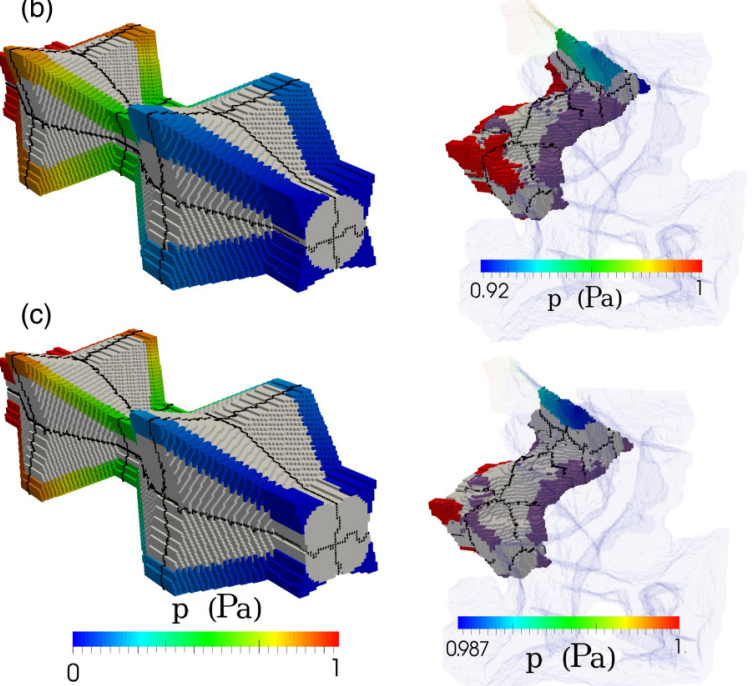

FIG. 5. Pressure fields for images generated by combining voxels of all corners of the generalized network into a single image, at different discretization levels. (a) Level 1, where the single-phase flow conductivities are computed. (b, c) Levels 2 and 3 that are obtained by excluding all the voxels inside maximal spheres with radius $r \geqslant R_{t}$ and $r \geqslant 0.7 R_{t}$, respectively. The excluded voxels are shown in light gray. The black lines highlight the boundaries between different corners in the images. In purple are shown the disconnected voxels from the inlet (left side) during single-phase flow simulations. The star-shaped geometry is shown on the left and a portion of the Bentheimer image on the right.

whole throat surface. $P_{p}$ is an average pressure at the adjacent pore center, computed by averaging the pressure of faces shared between voxels of different half-throats comprising the pore. This averaging is compatible with the formulation used in the network flow simulator, which computes the pressures at the pore centers. $Q$ is the volumetric flow rate passing through the corner at the throat surface and $\mu$ is the fluid viscosity used in the direct simulations.

A high resolution image is required for an accurate parametrization of corners, especially at discretization level 3 where the corner thickness is small. When a throat, or more specifically a corner, is not resolved for a given image resolution (voxel size), the corner voxels can become disconnected for one or more of the corner levels 2 and 3, and the computed conductivity for that corner level will be zero (see Fig. 5). In reality, however, the corner might be connected through subresolution crevices. The uncertainty related to the subresolution features of the 3D image could be added during flow simulation and is a subject for future work. 
An alternative approach for computing corner conductivities is to derive correlations from direct simulations on synthetic or real geometries. This approach can be considered as a faster method for estimating single- and two-phase properties of the void-space image without performing direct singlephase flow simulation, which requires high-performance computing: for instance each of our single-phase flow simulations on $1000^{3}$ voxel images, run in parallel on 32 processors at a clock speed of $2 \mathrm{GHz}$, took approximately $10 \mathrm{~h}$ of computational time, and required $250 \mathrm{~GB}$ of memory. In Appendix D, we have presented a set of simple correlations, which can be used as an alternative to direct simulations. In the following sections, we use this approach to evaluate the improvements achieved by the use of direct simulations for the computation of the corner conductivities. This algorithm, using correlations to compute corner conductivities, is called GNMCrl in this paper.

The parameters required for flow simulation are exported as tabulated data for each discretization level of the corners composing the generalized network model (GNM). These parameters include: (a) radius of the discretization level used in excluding the voxels in the pore and throat centers $\left(R_{i}, i=\right.$ 1-3); (b) unit vectors in the direction of the corner medial axis curves; (c) areas of the intersection of throat surfaces with the corner voxels at each level $\left(A_{i}\right)$; (d) volume of voxels at each level $\left(V_{i}\right)$; and (e) their flow conductivity $\left(g_{i}^{q}\right)$ obtained using direct single-phase flow simulation, Eq. (1).

We also considered electrical properties. In this paper, however, we use the correlations presented in Appendix D to compute the electrical properties and use direct simulation of formation factor (see Appendix C) to validate them.

Overall, the algorithm described above leads to a segmentation of the 3D image into half-throat corners as the main building blocks of the generalized network model. Each corner is described using the tabulated data extracted from the 3D images at the three discretization levels, as illustrated in Fig. 1. The parameters used to describe each level are cumulative: for instance, each level volume includes the volume of the voxels of the subsequent discretization levels. This implies that the volume of voxels in the discretization level 1 is the same as the corner total volume. Every corner, and hence every voxel, is assigned to both a pore and a throat. Here we define the pore volume as the volume of all the voxels associated with a pore. We also define a throat volume, which is the volume of all the voxels comprising the corners associated with the throat. Furthermore, each throat cross-sectional area is the sum of the cross-sectional area of the corners at level 1 . The total pore volume is the volume of all the pores; this is the same as the volume of all the throats and the same as the volume of all the corners.

The corners on opposite sides of a throat surface can be grouped to form a full corner. All the corners of the same throat can be further grouped to construct the whole throat. This grouping is used to compute a volume and a conductivity for each throat and to compute the pore and throat entry pressures during two-phase flow simulations, for instance. Each corner is also uniquely associated with a pore and is assumed to be connected (provide a path for layer flow) to its adjacent corners belonging to the same pore [78].

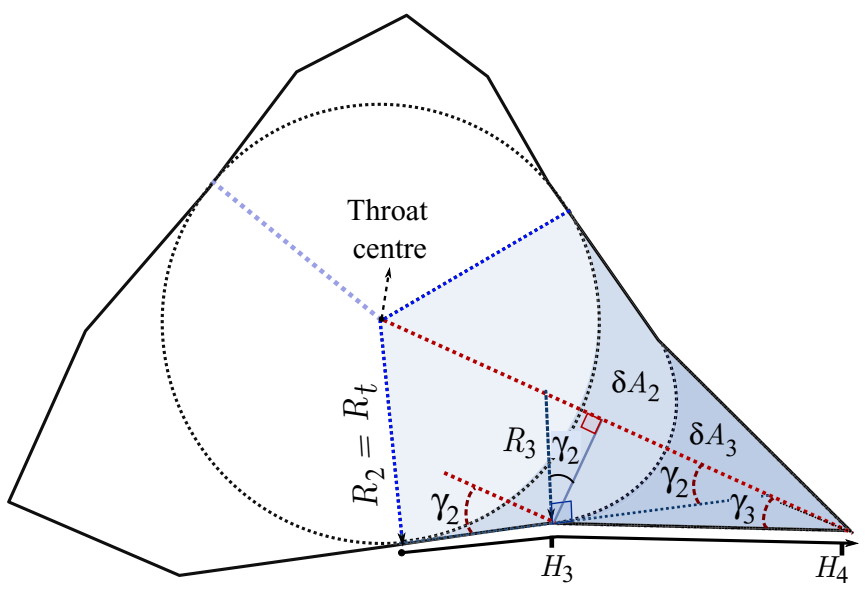

FIG. 6. An illustration of the parameters used to describe the shape of corners in the throat axial plane, including the radius of corner levels $\left(R_{i}\right)$ for levels $i=2-3$, their cross-sectional areas ( $\left.\delta A_{i}=A_{i}-A_{i+1}\right)$, corner half angles $\left(\gamma_{i}\right)$ and the corner depths measured along their perimeter $\left(H_{i}\right)$.

\section{SHAPES OF NETWORK NETWORK ELEMENTS}

In the following, we describe the explicit shape of the corners used to describe the pore space in the generalized network model.

A cross-section of a throat at the throat surface is shown in Fig. 6, comprising - in this case-three corners. The shape of this cross-section can be reconstructed from the extracted corner cross-sectional area and inscribed radius at levels 2 and 3 , for each of the corners. In this paper, we assume that the inscribed radius changes linearly between these two discretization levels. The inscribed radius and the cross-sectional area of each level can be used to compute two additional geometrical parameters for each level, namely corner half-angle $\left(\gamma_{i}\right)$ and corner depth measured along its edge $\left(H_{i}\right)$ :

$$
\begin{gathered}
\gamma_{i}=\sin ^{-1}\left(\frac{\cos \gamma_{i}+\gamma_{i} \sin \gamma_{i}}{\delta A_{i} / \delta R_{i}^{2}+\pi / 2}\right) \quad i=1-3, \\
H_{i+1}=H_{i}+\left(R_{i}-R_{i+1}\right) / \tan \gamma_{i}, \quad i=1-3,
\end{gathered}
$$

where, as before, $i$ labels the discretization level. $\delta A_{i}=A_{i}-A_{i+1}$ and $\delta R_{i}^{2}=R_{i}^{2}-R_{i+1}^{2}$. Note that these are implicit equations that have to be solved iteratively. These parameters can be used to track the interfaces of wetting layers residing in the crevices of the void space. They are also used to evaluate the accuracy of the network extraction workflow in the following sections.

We also need to define an explicit geometry away from the throat surface. In the axial cross-section, extra areas are added in the center to accommodate the change in the inscribed radius between the throat surface and the pore center. Figure 7 shows the cross-section of a corner at the pore center. The corner half angle $\left(\gamma_{1}\right)$ and depth $\left(H_{1}\right)$ for the added area are obtained using Eqs. (2) and (3) but from the corner cross-sectional areas computed at the pore center. Note that we assume the shape of the segments in the discretization levels 2 and 3 do not change as we move away from the throat surface. Furthermore, we assume that the 


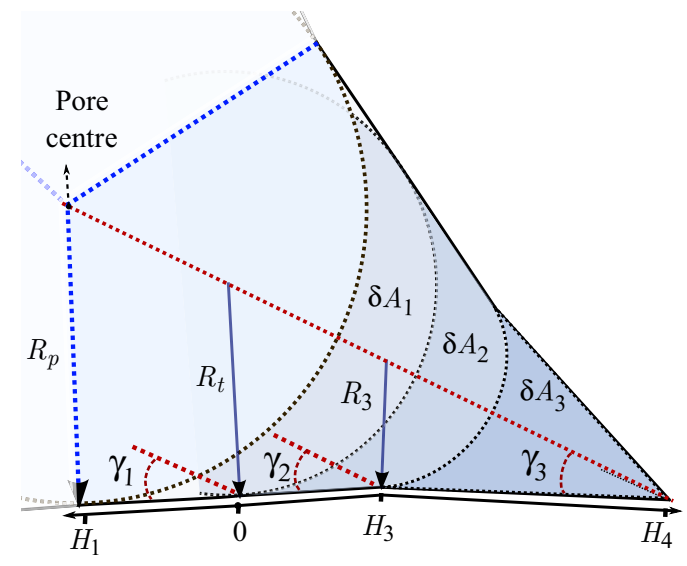

FIG. 7. An illustration of the a parameters used to describe the shape of corners at the pore center. Compared to the corner crosssection in Fig. 6, extra areas are added in the center to account for the expansion of the cross-section from the throat surface to the pore center. The corner half angle $\gamma_{1}$ and depth $H_{1}$ are introduced to account for this expansion.

distance of these levels from the throat line increases linearly, as illustrated in Fig. 1(d) in the corner sagittal plane.

The corner half-angles and depths used to reconstruct the shape of network elements are needed for multiphase flow simulation for tracking the location of fluid interfaces and, later in this paper, to construct a distance map distribution.

\section{VALIDATION}

The aim of this section is to show that the generalized network description of the void space obtained from our network extraction algorithm retains the geometry and properties of individual elements that are important for accurate multiphase flow simulations, such as effective corner angles and conductivities.

We first use synthetic geometries with predefined corner angles to evaluate the performance of the network extraction algorithm. Then we compute the statistics of the generated networks from micro-CT images of a set of porous rocks. The statistics presented include the pore and throat size distributions, corner angle distributions, average pore and throat sizes and average coordination number, as well as single-phase flow permeability and formation factors. Finally, to evaluate the accuracy of the network extraction algorithm on the micro-CT images, we compute the distribution of the distance-map from the extracted GNM and compare it with the distance map distribution obtained from the original 3D image. We also compare our results with conventional network models (CNM) generated using the maximal-ball algorithm [59].

\section{A. Synthetic geometries}

A set of four synthetic geometries are used to validate the network extraction workflow: two star-shaped geometries and two with triangular cross-sections. Each geometry is composed of two pores and three throats that connect the pores to each other and to the inlet and outlet boundaries; see Fig. 8. The shape of the pore and throat cross-sections (in their axial plan)

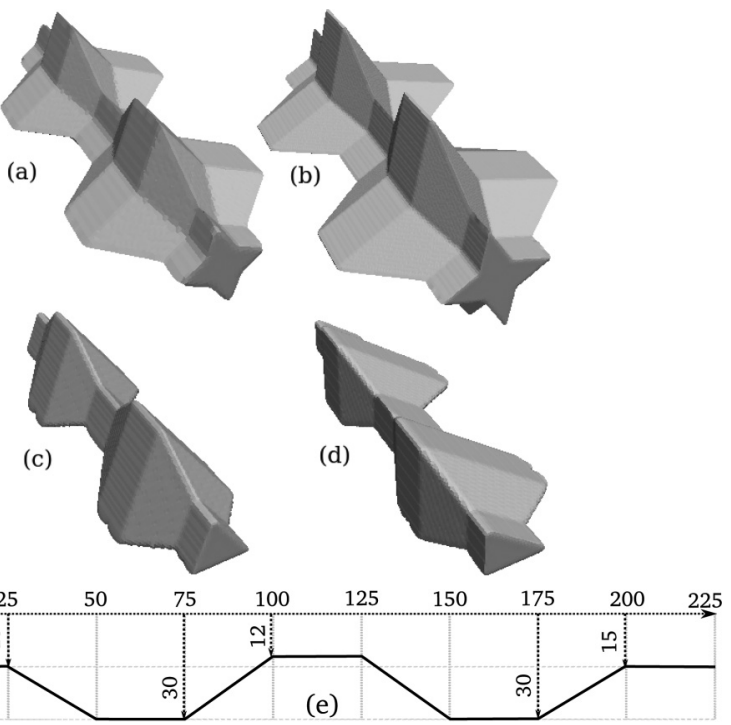

FIG. 8. Synthetic geometries used for the validation of the network extraction workflow: star-shaped geometries with cornerangles, $2 \gamma$, of (a) 60 and (b) 45 degrees and triangular geometries with corner angles of (c) 60-60-60 and (d) 40-60-80 degrees. (e) A plot of of their inscribed radius $\left(R_{i}\right)$ along the length of the void-space $(x)$, for all of the geometries.

remains constant, while their inscribed radius varies linearly between the pores and throats, as presented graphically in Fig. 8(e).

The OpenSCAD [85] software is used to create 3D surfaces representing the solid-walls of the synthetic geometries. These surfaces are then used to generate voxelized 3D volume images, similar to micro-CT images at a resolution of $R_{p} / \delta x=48$, where $\delta x$ is the voxel size. The contraction ratio for the middle throat is 2.5 , this leads to $R_{t} / \delta x=19.2$ at the throat. The images are then coarsened by factors of $2,4,8$, and 16 to study the convergence behavior of the network extraction workflow and compare it with direct numerical simulation (DNS) results. Note that the voxel values assigned to the coarse image voxels are the median of the values of its smaller composing voxels, which implies that the flow domain is not exactly the same between the images. Therefore, the results of this study can be considered as a characterization of both the errors associated with the voxelized representation of the geometries as well as the numerical errors introduced because of the approximations made during the modeling workflow.

Figures 9, 10, and 11 show the effect of image resolution on the accuracy of the predicted corner angles of the middle throat and the single-phase flow electrical resistance and permeability of the whole flow domain.

These results show that the generalized network extraction workflow predicts the single-phase permeability and formation factor accurately when the throat is resolved (with more than 4 voxels along its radius). This is not a surprise though: the GNM conductivities are computed using direct simulations. Moreover, these results confirm that the correlations used in GNMCrl produce acceptable results too.

The conventional network model representation does not accurately reproduce the conductivities and the corner angles 


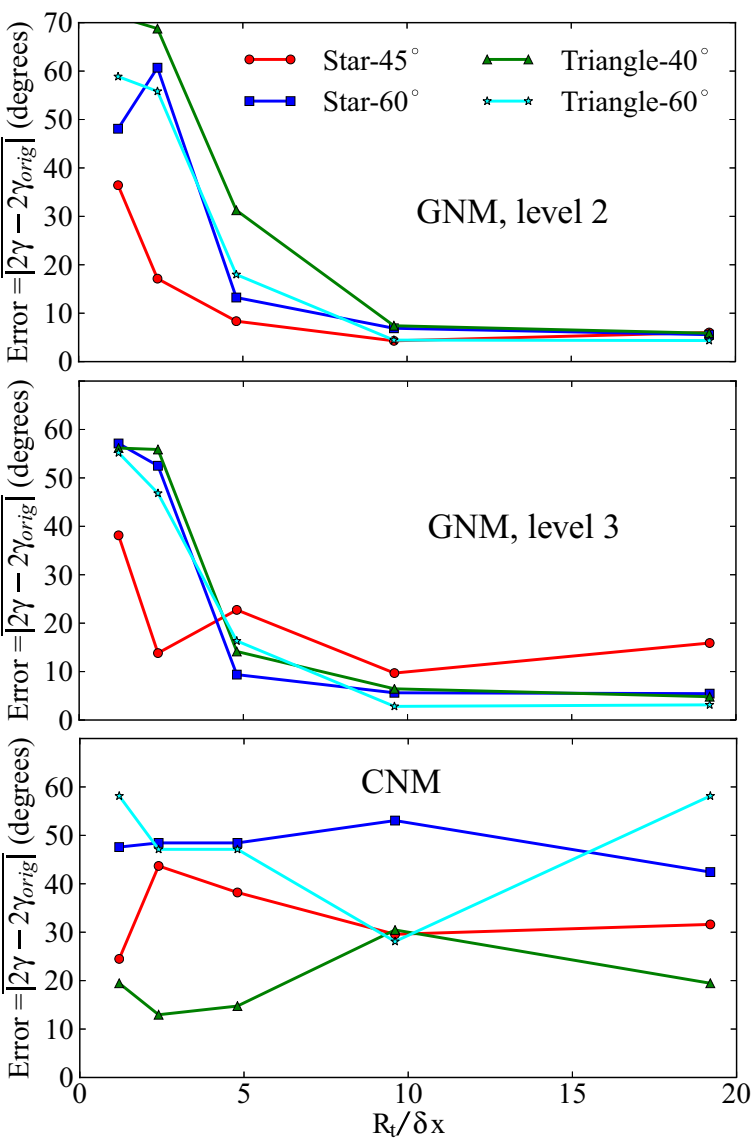

FIG. 9. Convergence tests for the computed corner angles using the generalized network model (GNM) at the two discretization levels, 2 and 3 , and using the conventional network model (CNM).

of the original geometry, even for the case of triangular pores, which are compatible with the shape of the pores chosen in the network model. The reason for this is that a shape factor cannot be used to define the three corner half angles $\left(\gamma_{c}, c=1-3\right)$ uniquely. See Appendix A for a discussion of the free parameters in the conventional network models, which then lead to uncertainties and errors in predicted properties [73]. The angles are assigned at random and hence will not necessarily represent the underlying geometry. Furthermore, the calculation of the shape uses the surface area between void and solid: with a voxelized stair-step discretization of the pore space, this leads to errors even for an infinitively refined image. This then means that the absolute permeability can be poorly estimated and does not converge to the correct value on grid refinement as shown in Fig. 11.

Overall, these results show that throats should be imaged at a resolution of at least $R_{t} / \delta x>5$ for the computed corner angles and absolute permeabilities to be predicted accurately using the generalized network model. The formation factors are, however, predicted accurately even for $R_{t} / \delta x>3$, which is expected since the value is less sensitive to the estimated throat radius. Further refinement in the generalized network model is needed to assign the corner angles more consistently when the throat is under resolved.

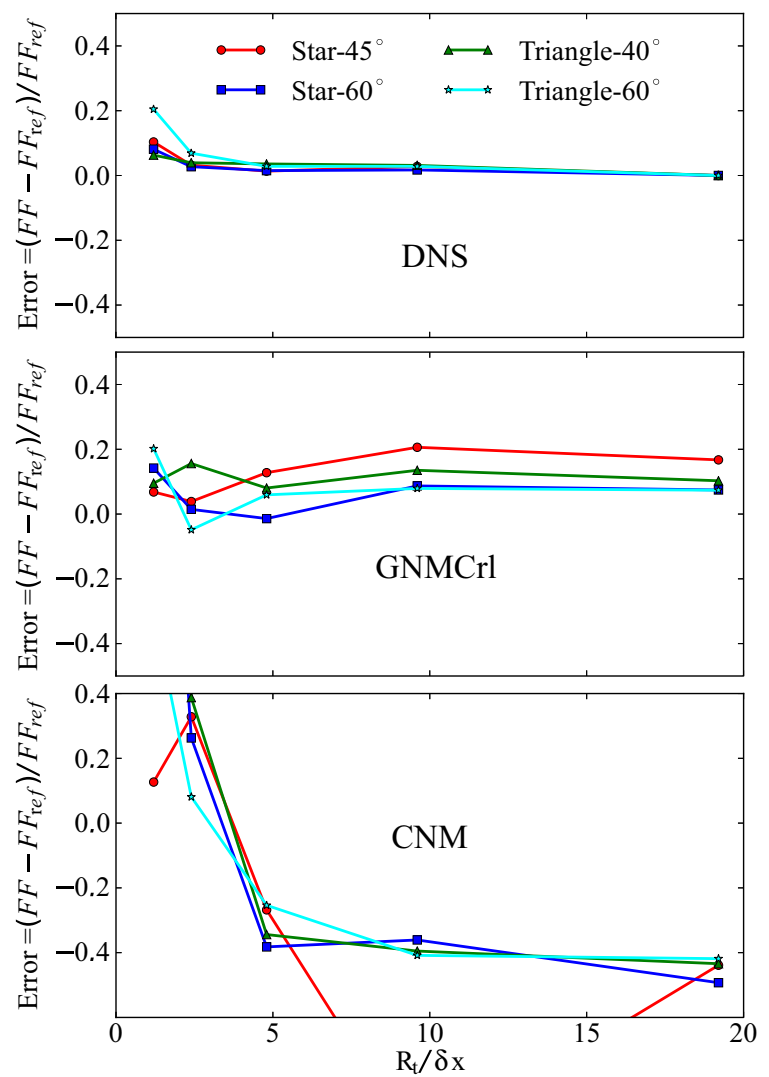

FIG. 10. Convergence tests for formation factors computed using the DNS, GNMCrl, and CNM formulations.

\section{B. Micro-CT images}

In this section, we present a comparison of the statistics of the network model that are relevant to flow simulations: coordination number, contraction ratio, and single-phase electrical resistivity and permeability. Figure 12 shows visualizations of extracted networks from four rock images: a Bentheimer sandstone image, provided by iRock Technologies [86], a Doddington sandstone, and Estaillades and Ketton carbonates that were obtained using the micro-CT scanning facilities at Imperial College London [87]. The images are segmented using a watershed segmentation algorithm using Avizo image processing software; see Ref. [88] for the technical details. The Bentheimer, Doddington, and Estaillades images have been previously analysed by Bijeljic et al. [80,89] and Alhashmi et al. [90]. A summary of the image properties are presented in Table I.

The probability density (PDF) of any network element parameter, $\varphi\left(=R_{p}, R_{t}, \gamma, \ldots\right)$, and its average value, $\bar{\varphi}$, are obtained arithmetically as well as using a weighted algorithm:

$$
\begin{gathered}
\operatorname{PDF}_{w}(\varphi)=\frac{\sum_{\varphi-\delta \varphi / 2}^{\varphi+\delta \varphi / 2} w_{\varphi}}{\delta \varphi \sum_{\varphi=-\infty}^{\infty} w_{\varphi}}, \\
\bar{\varphi}=\sum_{-\infty}^{\infty} \varphi \operatorname{PDF}_{w}(\varphi) .
\end{gathered}
$$

Choosing a weight of $w=1$ leads to the arithmetic average and the probability density function. It can be viewed as a 

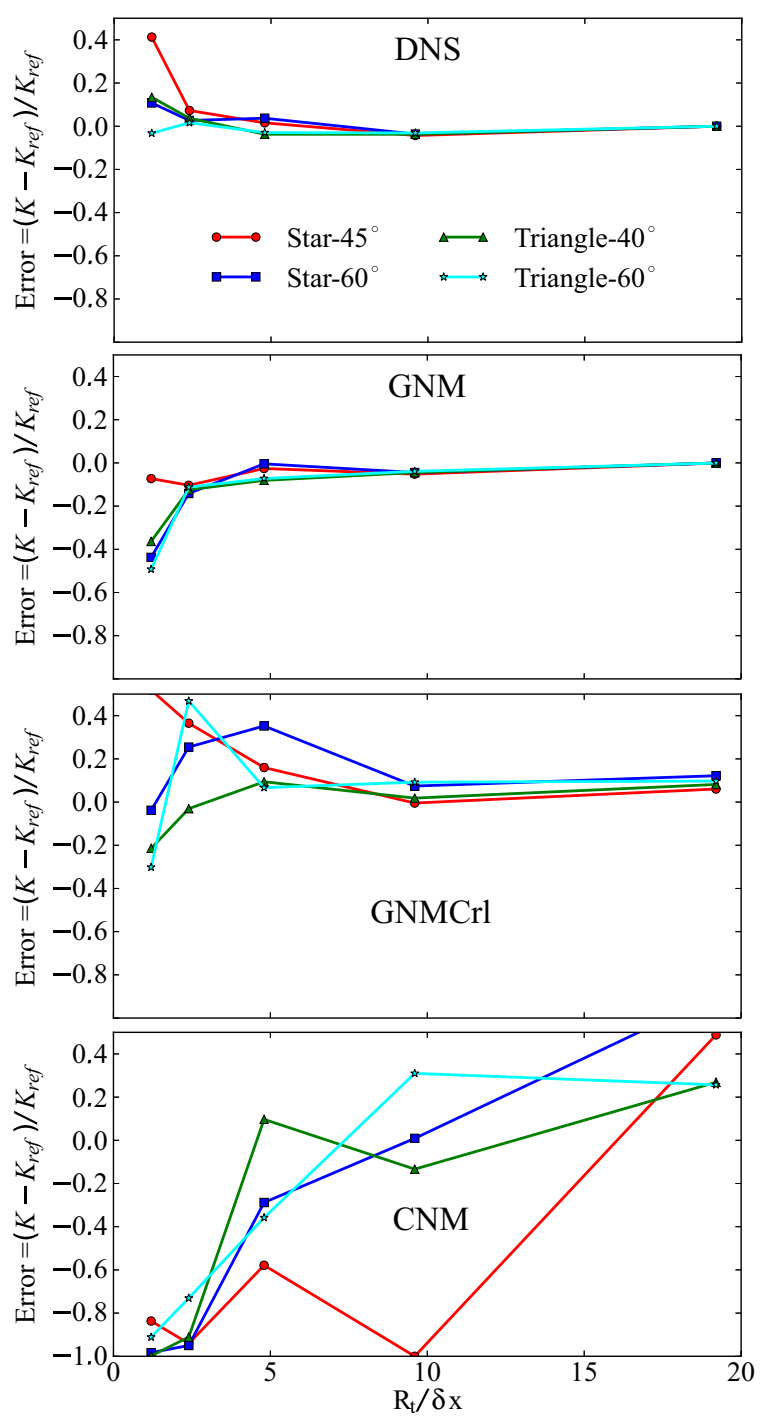

FIG. 11. Convergence of computed absolute permeability by increasing image resolution. Four methods are used direct numerical simulation (DNS) using the finite volume method, generalized network model (GNM), the generalized network model with conductivities computed using correlations (GNMCrl) presented in Appendix D, and a conventional network model (CNM).

measure of the frequency of elements with $\varphi$ as the parameter. The computed statistics using this approach can be sensitive to the filtering applied as a result of network extraction, since removing small unresolved elements from the network can have a big effect on the statistics. Choosing the weight, $w$, as the volume of the elements will produce a measure of the frequency of points or voxels that belong to network elements with $\varphi$ as their parameter. This choice of weighting will emphasize the bigger pores and throats - that can have more contribution to fluid volume and possibly flow conductivityand hence the distribution and the average values shift toward the parameters of the elements with larger volumes.

Figures 13 presents the distribution of pore and throat radii, using the volume-weighted approach $\left(w=V_{p}\right.$, and $w=V_{t}$, respectively, where $V_{p}$ is the pore volume, the volume of all the voxels associated with a pore, and $V_{t}$ is the volume of the voxels

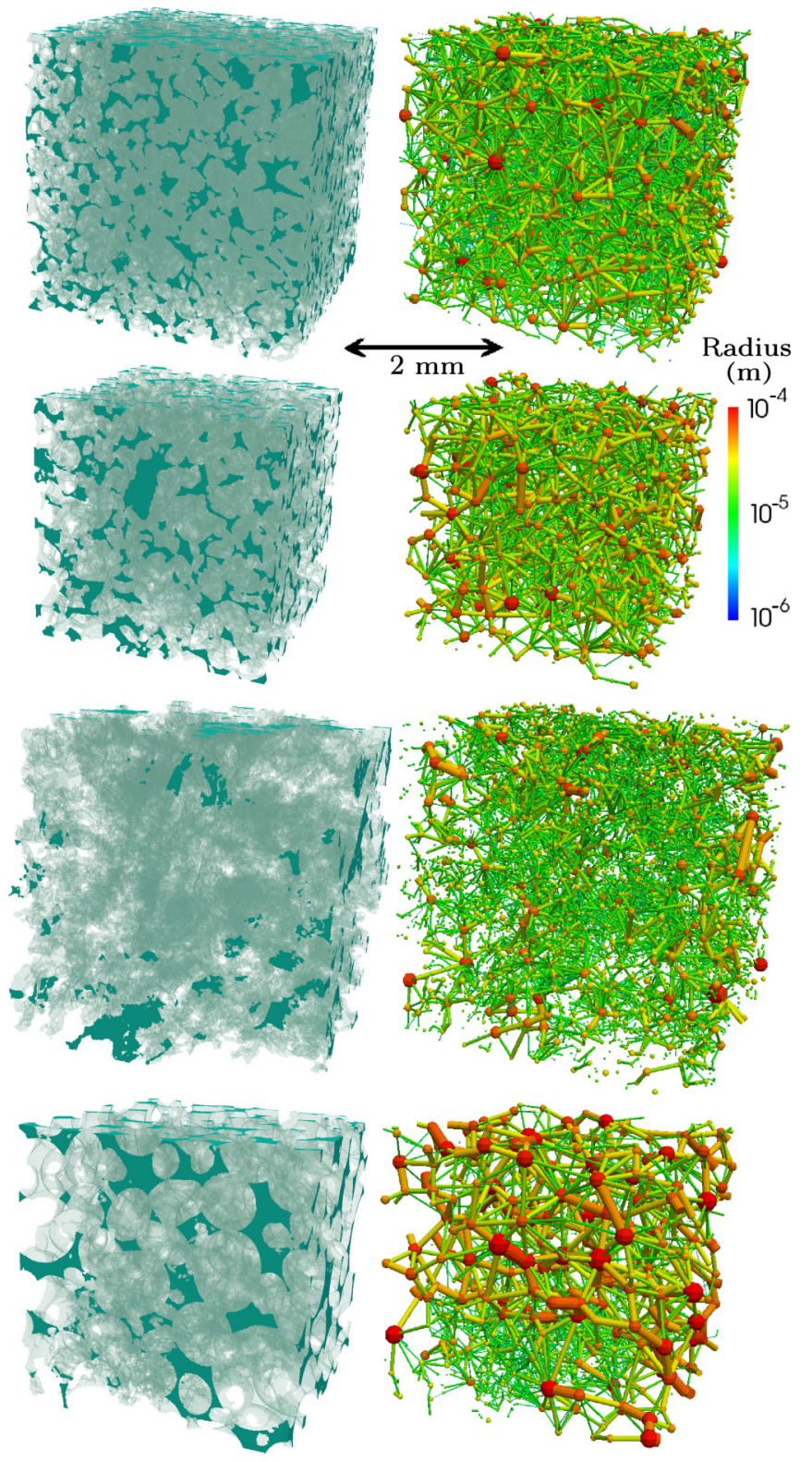

FIG. 12. Micro-CT images (left column) and a ball and stick visualization of the extracted networks (right column)—where throats are rendered as a cylinders and pores as spheres with radii proportional to their inscribed radii-for Bentheimer sandstone (1st row), Doddington sandstone (2nd row), and Estaillades (3rd row) and Ketton (4th row) carbonates.

belonging to the throat $t$ ). Figure 14 shows the distribution of the corner half-angles $(\gamma)$ at the corner discretization levels 2 and 3 .

Table I presents a summary of the average properties of the extracted networks, including averages of pore and throat radii, corner angles, contraction ratios, and coordination numbers, along with the computed absolute permeability and formation factor of the networks. We have compared our results with direct numerical simulation (DNS) and conventional network models (CNM) extracted using the maximal-ball algorithm [59]. 

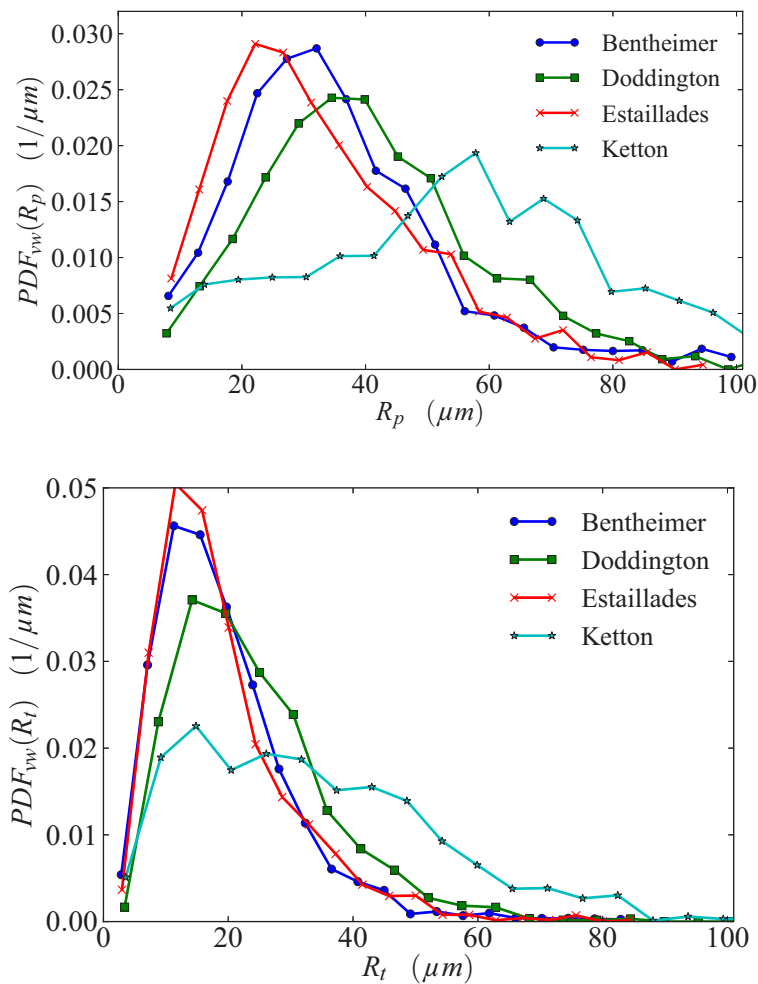

FIG. 13. Pore radius $\left(R_{p}\right)$ and throat radius $\left(R_{t}\right)$ distributions computed for the four micro-CT images, obtained using Eq. (4) with $w=V_{p}$ and $w=V_{t}$ as the weights, respectively.
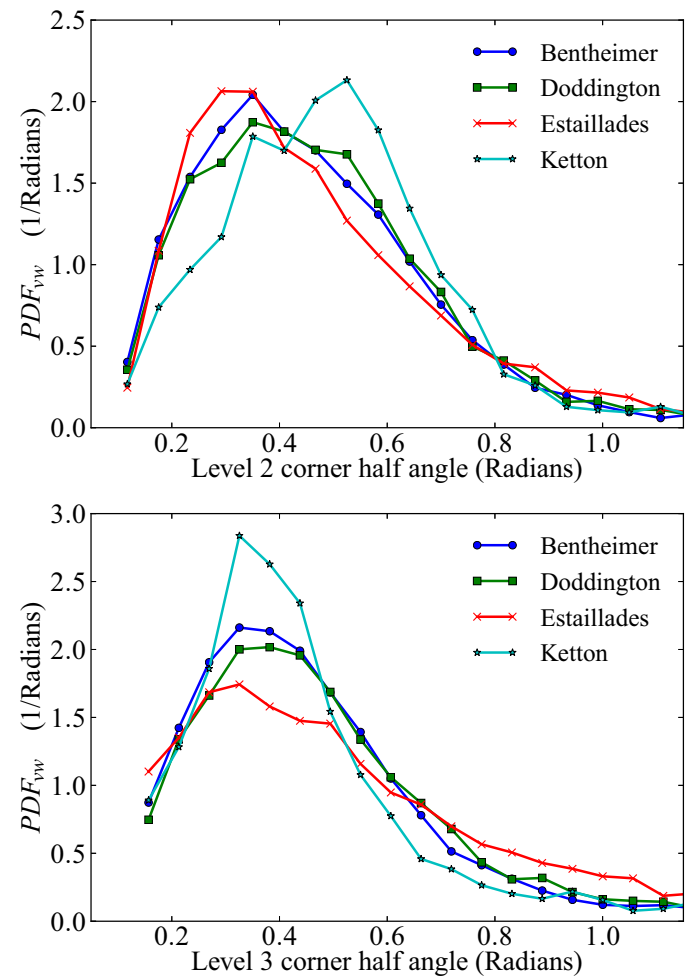

FIG. 14. Corner angle distributions for the four rock images, computed for levels 2 and 3 , corresponding to voxels outside maximal spheres with radius $r>R_{t}$ and $r>0.7 R_{t}$, using Eq. (4) with $w=V_{2}-V_{3}$ and $w=V_{3}$ as the weights, where $V_{2}$ and $V_{3}$ are the corner volumes at levels 2 and 3 , respectively.
TABLE I. Prediction of single-phase properties using the generalized network model (GNM) that matches the direct numerical simulation (DNS) results. The results are compared with a conventional network model (CNM) and the generalized network model with correlations (GNMCrl). Different measures of average properties of the networks are also presented. $w$ represents the weight used in Eqs. (4) and (5) to compute the average properties. $V_{p}, V_{t}, V_{2}$, and $V_{3}$ are the volume of pores, throats, corners at levels 2 and level 3 , respectively.

\begin{tabular}{lccccc}
\hline \hline Image & & Bent. & Dodd. & Esta. & Kett. \\
\hline size (voxels) & & $1000^{3}$ & $1000^{3}$ & $1000^{3}$ & $1000^{3}$ \\
voxel size $(\mu m)$ & & 3.003 & 2.693 & 3.311 & 3.000 \\
Porosity & & 0.216 & 0.194 & 0.109 & 0.132 \\
Absolute & DNS: & 3.55 & 3.76 & 0.218 & 5.91 \\
permeability & GNM: & 3.35 & 3.63 & 0.21 & 5.75 \\
$\left(\times 10^{-12} m^{2}\right)$ & GNMCrl: & 3.12 & 3.12 & 0.167 & 5.31 \\
& CNM: & 2.46 & 3.19 & 0.114 & 4.94 \\
Formation & DNS: & 15.5 & 20.0 & 166 & 24.2 \\
factor & GNMCrl: & 14.6 & 21.3 & 172 & 24.5 \\
& CNM: & 9.94 & 12.4 & 130 & 15.6 \\
Average number of corners & & & & \\
$w=1$, & GNM: & 1.70 & 1.73 & 1.42 & 1.6 \\
& CNM: & 3.002 & 3.005 & 3.004 & 3.003 \\
$w=V_{t}$, & GNM: & 2.32 & 2.39 & 2.23 & 2.46 \\
& CNM: & 3.001 & 3.000 & 3.001 & 3.001
\end{tabular}

Average corner angle at level 2, $2 \gamma_{2}$ (degrees)

$\begin{array}{llcccc}w=1, & \text { GNM: } & 72 & 76 & 82 & 76 \\ w=V_{2}-V_{3}, & \text { CNM: } & 30.0 & 30.1 & 30.1 & 30.0 \\ & \text { GNM: } & 54 & 56 & 56 & 59 \\ \text { CNM: } & 11.7 & 12.6 & 12.3 & 12.2\end{array}$

Average corner angle at level 3, $2 \gamma_{3}$ (degrees)

$w=1$,

GNM

8290

$105 \quad 85$

$w=V_{3}$,

GNM:

$53 \quad 57$

61

51

Average pore radius $(\mu \mathrm{m})$

$\begin{array}{llllll}w=1, & \text { GNM: } & 19.7 & 22.4 & 16.1 & 20.0\end{array}$

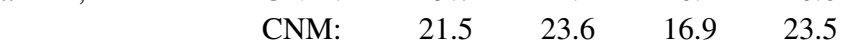

$w=V_{p}, \quad$ GNM: $\quad 35.5 \quad 41.4 \quad 33.0 \quad 55.2$

$\begin{array}{lllll}\text { CNM: } & 40.4 & 46.7 & 36.7 & 61.6\end{array}$

Average throat radius $(\mu m)$

$\begin{array}{llllll}w=1, & \text { GNM: } & 11.7 & 15.3 & 11.6 & 13.7\end{array}$

$\begin{array}{lllll}\text { CNM: } & 8.67 & 11.1 & 8.02 & 11.1\end{array}$

$w=V_{t}, \quad$ GNM: $\quad 19.2 \quad 24.1 \quad 19.2 \quad 33.3$

Average expansion ratio, $R_{p} / R_{t}$

$\begin{array}{llllll}w=1, & \text { GNM: } & 1.66 & 1.52 & 1.89 & 1.74\end{array}$

$\begin{array}{lllll}\text { CNM: } & 2.37 & 2.22 & 2.48 & 2.35\end{array}$

$w=V_{t}, \quad$ GNM: $\quad 2.19 \quad 2.03 \quad 2.01 \quad 2.21$

$\begin{array}{lllll}\text { CNM: } & 3.44 & 3.15 & 3.20 & 3.12\end{array}$

Average coordination number

$\begin{array}{llllll}w=1, & \text { GNM: } & 4.11 & 3.32 & 2.62 & 3.15\end{array}$

$\begin{array}{llllll} & \text { CNM: } & 3.78 & 3.05 & 4.85 & 3.66 \\ & \text { GNM: } & 8.18 & 7.277 & 7.37 & 7.29\end{array}$

\begin{tabular}{lllll} 
CNM: & 8.33 & 7.91 & 10.04 & 7.48 \\
\hline
\end{tabular}

There are several conclusions that can be made from these results about the accuracy of the network extraction methods: first, the generalized network model can reproduce direct single-phase flow simulation results when the conductivities 
are obtained from the analysis of direct simulations, although there is a slight mismatch, which can be attributed to the pressure and velocity averaging used to upscale the direct simulations, discussed in Sec. III, Stage 4. When not using direct simulations, the correlations used in the generalized network model (presented in Appendix D) can reproduce the single-phase results accurately, within $12 \%$ error for the results presented in Table I. The generalized network model extracts the number of corners from the image, which are usually fewer than that of the number of corners in the conventional network model, 3 on average.

The conventional network model produces results to within a factor of 2 for both permeability and formation factor compared to direct simulation. This is clearly worse than the generalized approach but better than seen for the simple geometries studied in the previous section. This apparent improvement in performance is because the boundary between pores and throats is adjusted to match the permeability obtained using direct simulation [59]. See Appendix A for further details. The problem here is that this is simply an adjustable tune of the model and provides no guarantee of the correct prediction of multiphase flow properties, or even reasonable predictions for other rock types.

The results presented in this section cannot be used as a proof that the extracted networks provide a good parametrization of other element properties which are relevant to multiphase flow through porous media (e.g., distribution of volumes between pores and throats and along the corner depths). To deal with this problem, in the next section, we use the distance-map distributions of the image and the extracted networks, as a measure of the distribution of the volume of the void space between pores and throats and along the crevices of the void space, to evaluate the accuracy of the network extraction workflows.

\section{Distance map distribution}

To estimate the accuracy of the coarse generalized network description of the micro-CT images presented above, we have computed the distance-map distribution of the network, by discretizing each corner into smaller conceptual thin elements around contour surfaces with a thickness of $\delta r$ and a distance of $r$ from the corner solid walls (hence parallel to the solid-walls of the corner; see Fig. 15). In essence, we now use the explicit representation of the network model in terms of equivalent corners to determine a distance map and compare this with the original image.

The distance distribution is obtained using Eq. (4), with the volume of the thin elements as the weight:

$$
w=A(r) \delta r,
$$

where $A(r)$, for the case of the generalized network model, is obtained from the tabulated corner depths $\left(H_{i}\right.$, Eq. (3)) and corner half angles $\left(\gamma_{i}\right.$, Eq. (2)):

$$
A(r)= \begin{cases}\frac{R_{t}+R_{p}}{2 R_{t}}\left(\delta H_{2} L_{2}+\delta H_{3} L_{3} \frac{R_{3}-r}{R_{3}}\right) & r<R_{3}, \\ \frac{R_{t}+R_{p}}{2 R_{t}} \delta H_{2} L_{2} \frac{R_{2}-r}{R_{2}} & R_{3}<r<R_{2}, \\ \frac{R_{p}-R_{t}}{\tan \left(\gamma_{1}\right)}\left(\frac{R_{p}-r}{R_{p}}\right)^{2} L_{h t} & R_{2}<r<R_{1},\end{cases}
$$

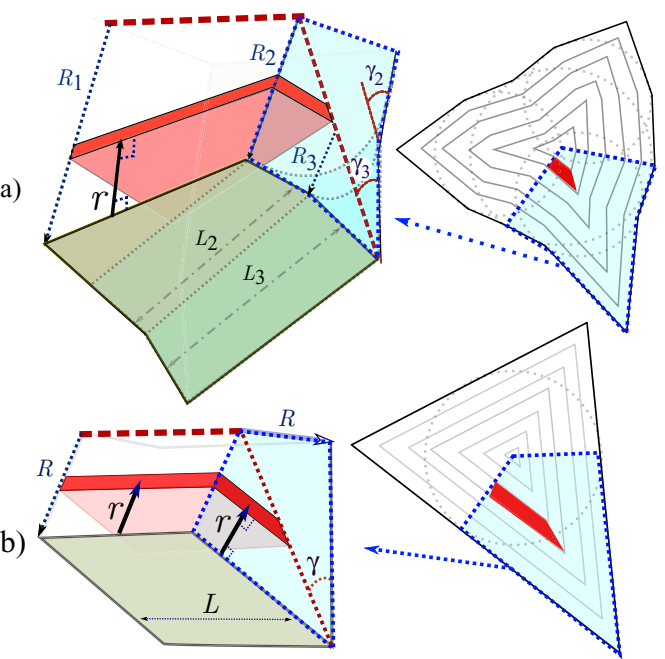

FIG. 15. An illustration of the network parameters used to obtain the distance map distribution for the (a) generalized and (b) the conventional network models. The thin elements highlighted in red have a uniform distance $(r)$ from the solid walls.

where $\delta H_{i}=H_{i+1}-H_{i}$. This equation is obtained assuming that the inscribed radius increases linearly between the pore center and the throat center (from $R_{1}=R_{p}$ to $R_{2}=R_{t}$ ).

$A(r)$ for corners of the conventional network model elements is computed as follows:

$$
A(r)=L \frac{R-r}{\tan (\gamma)},
$$

where $L$ is the pore or throat length, $R$ is their inscribed radius and $\gamma$ is the corner half angle.

Equation (4) is also used to obtain the distance map distribution from the 3D image, by using individual voxels as the elements over which the summations are performed. The results are presented in Fig. 16.

The distance map distributions show a good agreement between those obtained from the generalized network model and from the 3D image, while there is a clear mismatch with the distance map distribution obtained from the conventional network model. Distance map distributions are linked with the local geometry of the pores and throats-through Eq. (8) for the case of the conventional network model and through Eqs. (2), (3), and (7) for the case of the generalized network model. These results and the validation results presented for the case of synthetic geometries show that the generalized network model preserves the local geometry of the complex $3 \mathrm{D}$ images with a good accuracy.

\section{CONCLUSIONS AND FUTURE WORK}

We have presented a network extraction workflow which discretizes the pore space by subdividing it into pores bounded by throat surfaces, and further into half-throat corners. The network extraction algorithm is based on a medial-axis transformation-presented in terms of the hierarchy of maximal spheres located on the resulting medial surface. Hence, it preserves the local geometry of the void space down to the subpore scale, while filtering out surface-wall roughness. This 

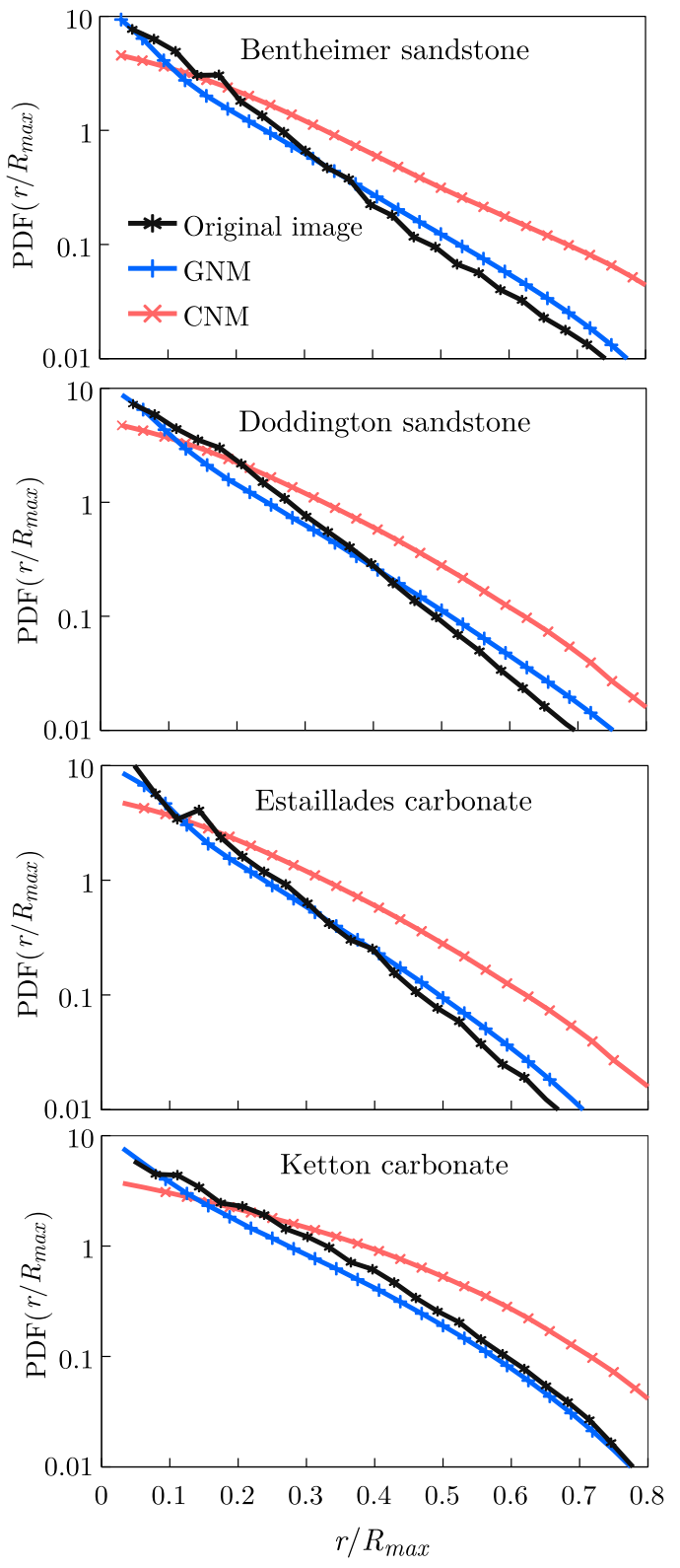

FIG. 16. Normalised distance map $\left(r / R_{\max }\right.$, where $R_{\max }$ is the radius of the biggest pore in the network) distributions for the four micro-CT images; the generalized network model results are compared with the conventional network model and the original image distance map distributions.

network description of the void space reduces the uncertainties associated with the oversimplification of the network elements and avoids the use of free parameters in conventional network models. Moreover, using direct simulations to quantify corner conductivities at different corner depths allows us to further minimize the errors associated with the relatively coarse discretization of the void space.

The quality of the extracted networks can be further improved by adjusting the parameters used during medial surface extraction for coarsening the medial surface and filtering surface roughness. Moreover, the description of the network elements can be further generalized to allow fracturelike elements making it possible to model multiphase flow through different classes of porous media.

The generalized description of the pore space can be used to predict their macroscopic properties, such as relative permeability and capillary pressure curves more accurately while retaining the computational efficiency of the conventional network models [78].

However, further work is needed to quantify the effect of different uncertainties in the modeling workflow. Examples of such uncertainties are image segmentation errors and the effect of features that are not resolved using micro-CT imaging, such as nanoscale pores and grain wall roughness and corner connectivity at scales below the image resolution. The coarse description of the void space allows efficient simulations, such as quasistatic capillary dominated two-phase flow simulations, to be performed quickly and hence can be used as a platform for quantification of the effect of these uncertainties in a practical simulation time.

\section{ACKNOWLEDGMENT}

The authors are grateful to TOTAL for financial support and permission to publish this work.

\section{APPENDIX A: FREE PARAMETERS IN THE CONVENTIONAL NETWORK MODELS}

As stated before, most of the conventional network element parameters, except inscribed radius $(R)$, are free parameters: they cannot be unambiguously determined from the analysis of a 3D image. The first free parameter is the boundary used to separate pores and throats. The assignment of the boundary can be chosen so that the network model predictions match some alternative data, for example, in single-phase flow simulations of absolute permeability on the same image [59]. The assignment of this boundary, in addition to affecting the computed pore and throat lengths (which is important for computation of single-phase flow properties), has an impact on the volume distribution between pores and throats [72] and the computed shape factor [39] (which is important for accurate prediction of fluid volumes and wetting layer conductivities in two-phase flow):

$$
G=A / P^{2},
$$

where $A$ is the pore or throat cross-sectional area computed by dividing the pore or throat volumes to their lengths. $P$ is their perimeter, which can be computed by dividing the pore and throat surface areas by their lengths [59].

The computed shape factors are then used to assign the shape of pores and throats during flow simulations: in the conventional network flow simulator used in this paper [42], if $G>0.079$, the shape is assumed to be a circular; otherwise, if $G>\sqrt{3} / 36$, a square is considered; and if $G \leqslant \sqrt{3} / 36$, it is assumed that the shape is triangular. One of the corner angles in triangular elements is randomly assigned, within its allowed range, while the two others are assigned to satisfy the geometrical constraint that the sum of the corner angles should be $180^{\circ}$ and the shape factor equation $[42,60]$.

Another major problem is the definition of shape factor given in Eq. (A1): it is sensitive to the level of surface wall roughness captured in the image, as illustrated in Fig. 17. 

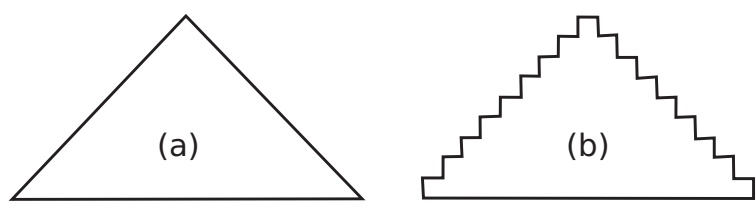

FIG. 17. An illustration of the fact that Eq. (A1) is sensitive to solid-wall roughness. The computed shape factor for a throat with a smooth triangular cross-section (a), $G=0.0429$, is very different from the shape factor for the same shape but with a stair-case roughness on two of its edges (b), $G=0.0278$.

\section{APPENDIX B: CALCULATION OF SINGLE-PHASE PERMEABILITY AND FORMATION FACTOR OF THE NETWORK MODEL}

To compute the absolute permeabilities, pressures are obtained at the pore centers and flow rates at the throats. They are obtained by solving the system of linear equations obtained by writing mass-balance equation for the pores:

$$
\sum_{t} Q_{t}=0,
$$

where $Q_{t}$ is the volumetric flow rate entering the pore from throat $t$, obtained using the Poiseuille equation for each throat:

$$
Q_{t}=g_{t}\left(P_{j}-P_{p}\right),
$$

where $P_{p}$ is the pore pressure and $P_{j}$ is the neighboring pore pressure, on the opposite side of the throat $t$.

$g_{t}$ is the throat transmissivity, which for the case of the conventional network model is computed using the same correlations as those presented in Valvatne and Blunt [42].

For the case of generalized network model with corner conductivities obtained from direct simulations, $g_{t}$ is computed as follows:

$$
1 / g_{t}=1 / g_{h t=1}+1 / g_{h t=2},
$$

where $g_{h t=1}$ and $g_{h t=2}$ are the two half-throat transmissivities obtained by summing the half-throat corner conductivities at discretization level $1, g_{1}^{c}$ :

$$
g_{h t}=\sum_{c} g_{1}^{c} .
$$

The two above equations assume that the conductivities are computed based on a single value for pressure at each pore or throat center. To be consistent with this assumption of the network flow simulator, we first compute an average value for pressure of pores and throats and use them to assign the same pressure drop for all the corners of the same half-throat. The average throat pressure is computed by averaging the pressure of all the throat faces as described in the main text. The average pore pressure is computed by averaging the pressures of voxel faces separating the half-throats of the pore.

Alternatively, the generalized network elements can be obtained using correlations, similar to the conventional network models but using corner parameters that are directly extracted from the 3D image. Appendix D presents the correlations that we have used to estimate the conductivity of corners at different discretization levels.

\section{APPENDIX C: DIRECT SIMULATION OF FORMATION FACTOR}

The direct numerical simulation results (DNS) for formation factors of the 3D images are obtained by solving the Laplace equation on a mesh with the image voxels as its grid-cells, using OpenFOAM [79] finite volume library, to compute the electric potential, $\phi^{e}$ :

$$
\nabla^{2} \phi^{e}=0
$$

subject to fixed-value boundary conditions of 1 and 0 at the inlet and outlets (left and right sides of the image), respectively, and zero-gradient boundary condition on the solid boundaries.

The electrical resistivity of the whole image can be calculated using the Ohm's law:

$$
\Delta \Phi^{e}=R I
$$

where $\Delta \Phi^{e}(=1)$ is the voltage across the flow domain, $I=$ $\sum_{f} \nabla \phi^{e} A_{f} / \rho_{w}^{e}$ is the total electrical flux entering the flow domain, $f$ counts over all the inlet voxel faces, $A_{f}$ is the voxel face area, $\nabla \phi^{e}$ stands for the electric field (gradient of electric potential) normal to the face, and $\rho_{w}^{e}$ is the fluid electrical resistivity. Finally, the formation factor $(F)$ is computed as follows:

$$
F=\frac{A_{D} \Delta \Phi^{e} / L}{\sum_{f} \nabla \phi^{e} A_{f}},
$$

where $L$ is the length of the image and $A_{D}$ is the image crosssectional area.

\section{APPENDIX D: CORRELATIONS FOR COMPUTING CORNER CONDUCTIVITIES}

In this section, we present a set of correlations that can be used to estimate the conductivity of the corner elements at different discretization levels. These correlations are used as a fast but approximate alternative to direct single-phase flow simulations. The conductivity of corner levels are first estimated assuming a uniform cross-sectional area, which are then corrected to accommodate the effect of expansion of the half-throat from its center to the neighboring pore center.

The corner electrical and flow conductivities $\left(g_{i}^{e}\right.$ and $g_{i}^{q}$, respectively), at discretization levels $i=2$ and 3 , are obtained using the following equations, which are approximations to the correlations used by Valvatne and Blunt [42] for corners of throats with equilateral triangle and square cross-sections:

$$
\begin{gathered}
g_{i}^{e}=A_{i} / L_{i}, \quad i=2,3, \\
g_{i}^{q}=\left(0.168-0.036 \gamma_{i}\right) R_{i}^{2} g_{i}^{e}, \quad i=2,3 .
\end{gathered}
$$

Then the conductivity of throat centers are estimated as follows:

$$
\begin{gathered}
g_{1}^{* e}=\frac{A_{1}-A_{2}}{L_{1}}, \\
g_{1}^{* q}=\frac{R_{2}^{2} g_{1}^{* e}}{8-4 A_{2} / A_{1}} .
\end{gathered}
$$

Finally, they are corrected for the effect of the expansion of the half-throat cross-sectional area between the throat center and 
the pores centers, assuming that the inscribed radius changes linearly:

$$
\begin{gathered}
g_{1}^{* * e}=g_{1}^{* e} R_{p / t}, \\
g_{1}^{* * q}=g_{1}^{* q} R_{p / t}^{3} /\left(1+\delta R_{p / t}+\delta R_{p / t}^{2} / 3\right),
\end{gathered}
$$

where $R_{p / t}=\frac{R_{p}}{R_{t}}$ and $\delta R_{p / t}=\frac{R_{p}-R_{t}}{R_{t}}$ are the expansion ratio and the relative expansion of the inscribed radius from the throat center to the pore center. Finally, the corner conductivities at level $i=2$ are added to these conductivities to obtain the level 1 (single-phase flow) conductivities:

$$
g_{1}^{e}=g_{1}^{* * e}+g_{2}^{e},
$$

$$
g_{1}^{q}=g_{1}^{* * q}+g_{2}^{q} .
$$

To obtain the corner conductivities at discretization levels $i=$ 2 and 3, an estimation of the corner length at these levels are needed. They are estimated as follows:

$$
L_{i}=\sqrt{L_{1}^{2}+\left(\frac{R_{p}-R_{t}}{\sin \gamma_{2}}\right)^{2}}, i=2,3 .
$$

The correlations presented above are obtained from a limited amount of simulations and available correlations in the literature. Further work is needed to better adjust their coefficients or to find more accurate alternatives.
[1] M. J. Blunt, Multiphase Flow in Permeable Media: A Pore-Scale Perspective (Cambridge University Press, Cambridge, 2017).

[2] M. Sahimi, Flow and Transport in Porous Media and Fractured Rock: from Classical Methods to Modern Approaches (John Wiley \& Sons, Hoboken, New Jersey, 2011).

[3] V. Cnudde and M. N. Boone, High-resolution X-ray computed tomography in geosciences: A review of the current technology and applications, Earth-Sci. Rev. 123, 1 (2013).

[4] M. J. Blunt, B. Bijeljic, H. Dong, O. Gharbi, S. Iglauer, P. Mostaghimi, A. Paluszny, and C. Pentland, Pore-scale imaging and modeling, Adv. Water Resour. 51, 197 (2013).

[5] P. Meakin and A. M. Tartakovsky, Modeling and simulation of pore-scale multiphase fluid flow and reactive transport in fractured and porous media, Rev. Geophys. 47, RG3002 (2009).

[6] P. M. Adler, Flow in simulated porous media, Int. J. Multiphase Flow 16, 691 (1990).

[7] P. M. Adler, C. G. Jacquin, and J. F. Thovert, The formation factor of reconstructed porous media, Water Resour. Res. 28, 1571 (1992).

[8] E. Jettestuen, J. O. Helland, and M. Prodanovic, A level set method for simulating capillary-controlled displacements at the pore scale with nonzero contact angles, Water Resour. Res. 49, 4645 (2013).

[9] A. Q. Raeini, M. J. Blunt, and B. Bijeljic, Direct simulations of two-phase flow on micro-CT images of porous media and upscaling of pore-scale forces, Adv. Water Resour. 74, 116 (2014).

[10] T. Ramstad, N. Idowu, C. Nardi, and P. E. Øren, Relative permeability calculations from two-phase flow simulations directly on digital images of porous rocks, Transp. Porous Media 94, 487 (2012).

[11] M. D. Jackson, P. H. Valvatne, and M. J. Blunt, Prediction of wettability variation and its impact on flow using pore- to reservoir-scale simulations, J. Pet. Sci. Eng. 39, 231 (2003).

[12] R. J. Held and M. A. Celia, Modeling support of functional relationships between capillary pressure, interfacial areas and common lines, Adv. Water Resour. 24, 325 (2001).

[13] C. Jia, K. Shing, and Y. C. Yortsos, Advective mass transfer from stationary sources in porous media, Water Resour. Res. 35, 3239 (1999).

[14] X. R. Zhou, N. Morrow, and S. Ma, Interrelationship of wettability, initial water saturation, aging time and oil recovery by spontaneous imbibition and waterflooding, SPE J. 5, 199 (2000).

[15] A. Raoof, H. M. Nick, S. M. Hassanizadeh, and C. J. Spiers, Poreflow: A complex pore-network model for simulation of reactive transport in variably saturated porous media, Comput. Geosci. 61, 160 (2013).

[16] J. Tansey and M. T. Balhoff, Pore network modeling of reactive transport and dissolution in porous media, Transp. Porous Media 113, 303 (2016).

[17] A. G. Yiotsis, A. K. Stubos, A. G. Boudouvis, and Y. C. Yortsos, A 2D pore-network model of the drying of singlecomponent liquids in porous media, Adv. Water Resour. 24, 439 (2001).

[18] D. Freitas and M. Prat, Pore network simulation of evaporation of a binary liquid from a capillary porous medium, Transp. Porous Media 40, 1 (2000).

[19] L. A. Dillard, H. I. Essaid, and M. J. Blunt, A functional relationship for field-scale nonaqueous phase liquid dissolution developed using a pore network model, J. Contam. Hydrol. 48, 89 (2001).

[20] J. P. Nogues, J. P. Fitts, M. A. Celia, and C. A. Peters, Permeability evolution due to dissolution and precipitation of carbonates using reactive transport modeling in pore networks, Water Resour. Res. 49, 6006 (2013).

[21] H. N. Man and X. D. Jing, Network modeling of wettability and pore geometry effects on electrical resistivity and capillary pressure, J. Pet. Sci. Eng. 24, 255 (1999).

[22] H. N. Man and X. D. Jing, Pore network modeling of electrical resistivity and capillary pressure characteristics, Transp. Porous Media 41, 263 (2000).

[23] H. N. Man and X. D. Jing, Network modeling of strong and intermediate wettability on electrical resistivity and capillary pressure, Adv. Water Resour. 24, 345 (2001).

[24] C. D. Tsakiroglou and M. Fleury, Pore network analysis of resistivity index for water-wet porous media, Transp. Porous Media 35, 89 (1999).

[25] M. Balhoff, D. Sanchez-Rivera, A. Kwok, Y. Mehmani, and M. Prodanović, Numerical algorithms for network modeling of yield stress and other non-newtonian fluids in porous media, Transp. Porous Media 93, 363 (2012).

[26] M. T. Balhoff and K. E. Thompson, A macroscopic model for shear-thinning flow in packed beds based on network modeling, Chem. Eng. Sci. 61, 698 (2006). 
[27] H. Kharabaf and Y. Yortsos, Pore network model for foam formation and propagation in porous media, SPE J. 3, 42 (1998).

[28] R. Bora, B. B. Maini, and A. Chakma, Flow visualization studies of solution gas drive process in heavy oil reservoirs with a glass micromodel, SPE Reserv. Eval. Eng. 3, 224 (2000).

[29] C. Du and Y. Yortsos, A numerical study of the critical gas saturation in a porous medium, Transp. Porous Media 35, 205 (1999).

[30] M. Jamiolahmady, A. Danesh, D. Tehrani, and D. B. Duncan, A mechanistic model of gas-condensate flow in pores, Transp. Porous Media 41, 17 (2000).

[31] X. Wang and K. Mohanty, Critical condensate saturation in porous media, J. Colloid Interface Sci. 214, 416 (1999).

[32] X. Wang and K. Mohanty, Pore-network model of flow in gascondensate reservoirs, SPE J. 5, 426 (2000).

[33] J. Carmeliet, F. Descamps, and G. Houvenaghel, A multiscale network model for simulating moisture transfer properties of porous media, Transp. Porous Media 35, 67 (1999).

[34] A. Ahmadi, A. Aigueperse, and M. Quintard, Calculation of the effective properties describing active dispersion in porous media: From simple to complex unit cells, Adv. Water Resour. 24, 423 (2001).

[35] B. Bijeljic and M. J. Blunt, Pore-scale modeling and continuous time random walk analysis of dispersion in porous media, Water Resour. Res. 42, W01202 (2006).

[36] C. Bruderer and Y. Bernabe, Network modeling of dispersion: Transition from taylor dispersion in homogeneous networks to mechanical dispersion in very heterogeneous ones, Water Resour. Res. 37, 897 (2001).

[37] A. Al-Futaisi and T. W. Patzek, Impact of wettability alteration on two-phase flow characteristics of sandstones: A quasistatic description, Water Resour. Res. 39, 1042 (2003).

[38] T. R. Lerdahl, P.-E. Øren, and S. Bakke, A predictive network model for three-phase flow in porous media, in Proceedings of the SPE/DOE Improved Oil Recovery Symposium, Tulsa, Oklahoma, 3-5 April, SPE-59311-MS (Society of Petroleum Engineers, 2000).

[39] P. E. Øren, S. Bakke, and O. J. Arntzen, Extending predictive capabilities to network models, SPE J. 3, 324 (1998).

[40] M. Piri and M. J. Blunt, Three-dimensional mixed-wet random pore-scale network modeling of two- and three-phase flow in porous media. II. results, Phys. Rev. E 71, 026302 (2005).

[41] D. S. Svirsky, M. I. J. van Dijke, and K. S. Sorbie, Prediction of three-phase relative permeabilities using a pore-scale network model anchored to two-phase data, in SPE Annual Technical Conference and Exhibition, SPE-89992-PA (Society of Petroleum Engineers, 2007), Vol. 10, pp. 527-538.

[42] P. H. Valvatne and M. J. Blunt, Predictive pore-scale modeling of two-phase flow in mixed wet media, Water Resour. Res. 40, W07406 (2004).

[43] S. Bryant and M. Blunt, Prediction of relative permeability in simple porous media, Phys. Rev. A 46, 2004 (1992).

[44] S. L. Bryant, P. R. King, and D. W. Mellor, Network model evaluation of permeability and spatial correlation in a real random sphere packing, Transp. Porous Media 11, 53 (1993).

[45] S. L. Bryant, D. W. Mellor, and C. A. Cade, Physically representative network models of transport in porous media, AIChE J. 39, 387 (1993).
[46] J. L. Finney, Random packings and the structure of simple liquids. I. The geometry of random close packing, Proc. R. Soc. London A: Math. Phys. Eng. Sci. 319, 479 (1970).

[47] S. Bakke and P. E. Øren, 3D pore-scale modeling of sandstones and flow simulations in the pore networks, SPE J. 2, 136 (1997).

[48] P. E. Øren and S. Bakke, Process based reconstruction of sandstones and prediction of transport properties, Transp. Porous Media 46, 311 (2002).

[49] P. M. Adler and J. F. Thovert, Real porous media: Local geometry and macroscopic properties, Appl. Mech. Rev. 51, 537 (1998).

[50] Z. Liang, M. A. Ioannidis, and I. Chatzis, Permeability and electrical conductivity of porous media from 3D stochastic replicas of the microstructure, Chem. Eng. Sci. 55, 5247 (2000).

[51] H. Okabe and M. J. Blunt, Pore space reconstruction using multiple-point statistics, J. Pet. Sci. Eng. 46, 121 (2005).

[52] J. Quiblier, A new three-dimensional modeling technique for studying porous media, J. Colloid Interface Sci. 98, 84 (1984).

[53] C. Tsakiroglou and A. Payatakes, Characterization of the pore structure of reservoir rocks with the aid of serial sectioning analysis, mercury porosimetry and network simulation, Adv. Water Resour. 23, 773 (2000).

[54] H. J. Vogel and K. Roth, Quantitative morphology and network representation of soil pore structure, Adv. Water Resour. 24, 233 (2001).

[55] H. Q. Zhao, I. Macdonald, and M. Kwiecien, Multiorientation scanning: A necessity in the identification of pore necks in porous media by $3 \mathrm{D}$ computer reconstruction from serial section data, J. Colloid Interface Sci. 162, 390 (1994).

[56] D. Wildenschild and A. P. Sheppard, X-ray imaging and analysis techniques for quantifying pore-scale structure and processes in subsurface porous medium systems, Adv. Water Resour. 51, 217 (2013).

[57] A. Al-Kharusi and M. J. Blunt, Multiphase flow predictions from carbonate pore space images using extracted network models, Water Resour. Res. 44, W06S01 (2008).

[58] F. Arand and J. Hesser, Accurate and efficient maximal ball algorithm for pore network extraction, Comput. Geosci. 101, 28 (2017).

[59] H. Dong and M. J. Blunt, Pore-network extraction from microcomputerized-tomography images, Phys. Rev. E 80, 036307 (2009).

[60] T. W. Patzek, Verification of a complete pore network simulator of drainage and imbibition, SPE J. 6, 144 (2001).

[61] D. Silin and T. Patzek, Pore space morphology analysis using maximal inscribed spheres, Physica A: Stat. Mech. Appl. 371, 336 (2006).

[62] C. A. Baldwin, A. J. Sederman, M. D. Mantle, P. Alexander, and L. F. Gladden, Determination and characterization of the structure of a pore space from 3D volume images, J. Colloid Interface Sci. 181, 79 (1996).

[63] W. B. Lindquist, S. M. Lee, D. A. Coker, K. W. Jones, and P. Spanne, Medial axis analysis of void structure in threedimensional tomographic images of porous media, J. Geophys. Res.: Solid Earth 101, 8297 (1996).

[64] A. Rabbani, S. Jamshidi, and S. Salehi, An automated simple algorithm for realistic pore network extraction from microtomography images, J. Pet. Sci. Eng. 123, 164 (2014). 
[65] R. I. Al-Raoush and C. S. Willson, Extraction of physically realistic pore network properties from three-dimensional synchrotron x-ray microtomography images of unconsolidated porous media systems, J. Hydrol. 300, 44 (2005).

[66] C. H. Arns, M. A. Knackstedt, W. V. Pinczewski, and N. S. Martys, Virtual permeametry on microtomographic images, J. Pet. Sci. Eng. 45, 41 (2004).

[67] M. A. Ioannidis and I. Chatzis, On the geometry and topology of 3D stochastic porous media, J. Colloid Interface Sci. 229, 323 (2000).

[68] Z. R. Liang, C. P. Fernandes, F. S. Magnani, and P. C. Philippi, A reconstruction technique for three-dimensional porous media using image analysis and fourier transforms, J. Pet. Sci. Eng. 21, 273 (1998).

[69] D. A. Coker, S. Torquato, and J. H. Dunsmuir, Morphology and physical properties of Fontainebleau sandstone via a tomographic analysis, J. Geophys. Res.: Solid Earth 101, 17497 (1996).

[70] W. B. Lindquist and A. Venkatarangan, Investigating 3D geometry of porous media from high resolution images, Phys. Chem. Earth, Part A: Solid Earth Geodesy 24, 593 (1999).

[71] P. Spanne, J. F. Thovert, C. J. Jacquin, W. B. Lindquist, K. W. Jones, and P. M. Adler, Synchrotron Computed Microtomograhy of Porous Media: Topology and Transports, Phys. Rev. Lett. 73, 2001 (1994).

[72] N. Idowu, C. Nardi, H. Long, P. E. Øren, and I. Bondino, Improving digital rock physics predictive potential for relative permeabilities from equivalent pore networks, in Proceedings of the International Symposium of the Society of Core Analysts, Napa Valley, CA, 16-19 September, SCA 2013-17 (2013).

[73] I. Bondino, G. Hamon, W. Kallel, and D. Kachuma, Relative permeabilities from simulation in 3D rock models and equivalent pore networks: Critical review and way forward, Petrophysics 54, 538 (2013).

[74] P. Giblin and B. B. Kimia, A formal classification of 3D medial axis points and their local geometry, IEEE Trans. Pattern Anal. Mach. Intell. 26, 238 (2004).

[75] M. Styner, G. Gerig, S. Joshi, and S. Pizer, Automatic and robust computation of 3D medial models incorporating object variability, Int. J. Comput. Vis. 55, 107 (2003).

[76] A. Lieutier, Any open bounded subset of $R^{n}$ has the same homotopy type as its medial axis, Computer-Aided Design 36, 1029 (2004).
[77] A. P. Sheppard, R. M. Sok, and H. Averdunk, Improved pore network extraction methods, in International Symposium of the Society of Core Analysts, SCA2005-20 (2005), pp. 21-25.

[78] A. Q. Raeini, B. Bijeljic, and M. J. Blunt, Generalized network modeling: Capillary-dominated two-phase flow-model description, arXiv:1707.02788 [physics.comp-ph].

[79] OpenFOAM, The open source CFD toolbox, http://www. openfoam.com (2016).

[80] B. Bijeljic, A. Raeini, P. Mostaghimi, and M. J. Blunt, Predictions of non-Fickian solute transport in different classes of porous media using direct simulation on pore-scale images, Phys. Rev. E 87, 013011 (2013).

[81] A. Q. Raeini, M. J. Blunt, and B. Bijeljic, Modelling two-phase flow in porous media at the pore scale using the volume-of-fluid method, J. Comput. Phys. 231, 5653 (2012).

[82] H. Dehghanpour, B. Aminzadeh, and D. A. DiCarlo, Hydraulic conductance and viscous coupling of three-phase layers in angular capillaries, Phys. Rev. E 83, 066320 (2011).

[83] A. Q. Raeini, B. Bijeljic, and M. J. Blunt, Numerical modeling of subpore scale events in two-phase flow through porous media, Transp. Porous Media 101, 191 (2014).

[84] C. Xie, A. Q. Raeini, Y. Wang, M. J. Blunt, and M. Wang, An improved pore-network model including viscous coupling effects using direct simulation by the lattice Boltzmann method, Adv. Water Resour. 100, 26 (2017).

[85] OpenSCAD, The programmers solid 3D CAD modeller, http://www.openscad.org (2016).

[86] iRock Technologies (2013), retrieved from http://www. irocktech.com.

[87] Imperial College London, Pore Scale Modelling group: Micro-CT images and networks, retrieved from http://www.imperial.ac.uk/engineering/departments/earthscience/research/research-groups/perm/research/pore-scalemodelling/micro-ct-images-and-networks (2017).

[88] M. G. Andrew, Reservoir-condition pore-scale imaging of multiphase flow, Ph.D. thesis, Imperial College London, 2014.

[89] B. Bijeljic, P. Mostaghimi, and M. J. Blunt, Insights into nonFickian solute transport in carbonates, Water Resour. Res. 49, 2714 (2013).

[90] Z. Alhashmi, M. J. Blunt, and B. Bijeljic, The impact of pore structure heterogeneity, transport, and reaction conditions on fluid-fluid reaction rate studied on images of pore space, Transp. Porous Media 115, 215 (2016). 